\title{
Benchmarking atomic data for astrophysics: Fe xI
}

\author{
G. Del Zanna \\ Department of Applied Mathematics and Theoretical Physics, University of Cambridge, Wilberforce road, Cambridge CB3 0WA, UK \\ e-mail: G.Del-Zanna@damtp.cam.ac.uk
}

Received 14 January 2010 / Accepted 4 February 2010

\section{ABSTRACT}

\begin{abstract}
High-resolution spectroscopic observations of the solar corona and laboratory measurements are used to review all the line identifications for Fe XI, from the EUV to the visible. The results of the atomic structure and scattering calculations are presented elsewhere, while detailed comparisons between observed and predicted wavelengths and intensities are discussed here. All the brightest EUV lines in the solar corona are now finally firmly identified. Several new identifications are proposed, in particular, coronal forbidden lines. The previously-known density-diagnostics are confirmed. New and important temperature diagnostics are presented, and the presence of blends highlighted.
\end{abstract}

Key words. atomic data - line: identification - Sun: corona - techniques: spectroscopic

\section{Introduction}

This paper is one in a series that aims to provide an assessment of available atomic data for the analysis of astrophysical spectra, by benchmarking them against all available experimental data. The approach is based on observations, and focuses on the brightest spectral lines that are observed in solar and/or laboratory spectra. The complexities of the benchmark method, and the available types of theoretical and experimental data that are normally available are described in Del Zanna et al. (2004) (Paper I).

Fe XI is an important ion for the solar corona because it produces very strong spectral lines that can in principle be used for plasma diagnostics and for instrument calibration. Lines of Fe XI have been recorded by all solar coronal missions (e.g. Skylab, SOHO), and are particularly important for the Hinode EUV Imaging Spectrometer (EIS, see Culhane et al. 2007), because many strong Fe XI lines fall at EIS wavelengths. The instrument covers two EUV wavelength bands (SW: 166-212 ̊; LW: 245-291 ^).

Because Fe XI is a complex ion, it took about five years to identify almost all the energies of the lowest configurations and to find a convergence between atomic calculations and experimental data. The atomic calculations are described in Del Zanna et al. (2010), while the description and assessment of the experimental data is provided in this complementary paper. One difficulty was that most energies were not known, and of those known, many conflicting identifications are present in the literature. This is partly because ab initio calculations are usually not good enough to match the observations and the predicted wavelengths are often inaccurate by a few $\AA$. An extensive analysis along the entire $\mathrm{S}$ I-like sequence was done, both in terms of atomic structure (see some results published in Del Zanna et al. 2010) and in terms of experimental data. Results pertaining to other ions will be published elsewhere. Another problem discussed at length in Del Zanna et al. (2010) is that a few important levels are strongly mixed, and it is difficult to find a good target that provides reliable oscillator strengths (hence reliable transition probabilities and excitation rates) for transitions from these levels. No previously-published work was found to contain accurate enough oscillator strengths. For details of some of the previous radiative and collisional calculations, and some comparisons, see Del Zanna et al. (2010).

Section 2 briefly describes the experimental data considered for this benchmark. Section 3 briefly describes the benchmark method. Section 4 presents a summary of the main results, while Sect. 5 discusses the details of the line identifications and the diagnostics. Section 6 draws the conclusions.

\section{Experimental data}

There is over a century of spectroscopic observations of the solar corona. However, very few solar observations had sufficient spectral resolution and were radiometrically calibrated in a way that is independent of the use of atomic data, making them directly usable for the benchmark.

Most Fe XI line identifications in the EUV have been made using laboratory measurements of B.C. Fawcett and the group at the Culham laboratories in the sixties and seventies. Prominent papers are Gabriel et al. (1966), Fawcett (1971), and Bromage et al. (1977). These laboratory spectra were not free of impurities, but had excellent resolution and are best for identifying lines that are formed in high-density plasmas. Some of the original plates and unpublished material obtained from B.C. Fawcett were used for the present assessment. More recently, a few papers based on laboratory spectroscopy (e.g. beam-foil and electron beam ion traps) have produced very useful spectral data for the identifications of Fe XI lines (see, e.g. Jupén et al. 1993; Träbert 1998).

\subsection{Solar EUV spectra}

Behring et al. (1972) published a line list based on an LASP rocket flight that observed the entire Sun in the 60-385 A region with high resolution (0.06 ̊). Behring et al. (1976) presented similar results, covering the $160-770 \AA$ range. In the latter paper (hereafter Be76), the wavelengths of the lines were accurately 
measured using higher orders and are still the best EUV wavelengths for many ions.

Malinovsky \& Heroux (1973) presented an integrated-Sun spectrum of medium resolution $(0.25 \AA)$, covering the $50-300 \AA$ range, that was taken with a grazing-incidence spectrometer flown on a rocket in 1969. The spectrum was photometrically calibrated and still is the best available spectrum in the EUV (150-300 $\AA$ ), in terms of radiometric calibration (10-20\% relative uncertainty). The published list of intensities provided by Malinovsky \& Heroux (1973) was not complete, so additional intensities for some weaker lines were obtained from their spectrum, by calibrating it in intensity.

Many other line lists have been published, but are normally from active region spectra where strong blending with high-temperature lines is present, so they are of limited use for benchmarking Fe XI. For example, the Goddard Solar Extreme Ultraviolet Rocket Telescope and Spectrograph (SERTS) has been flown several times since 1989 and has produced data of excellent spectral resolution, mostly of active regions. The SERTS-89 (Thomas \& Neupert 1994; hereafter TN94) covered the 170-225 $\AA$ range in second order and the 235-450 $\AA$ range in first order. The SERTS-97 (Brosius et al. 2000) covered the 300-353 A spectral region. The SERTS-95 spectra (Brosius et al. 1998b) covered the 171-225 $\AA$ band in second-order, and the 235-335 $\AA$ region in first order with excellent spectral resolution $(F W H M=0.03,0.05 \AA$ respectively). However, atomic data (CHIANTI version 1.01) were actually used by Brosius et al. (1998a) to calibrate the SERTS-95 spectra.

The SERTS-89 and SERTS-97 were radiometrically calibrated on the ground against primary standards and in theory could be used for the benchmark. However, major problems are found in the the calibration of the second-order lines as discussed for example in Del Zanna (1999). Doubts about the calibration in the 400-450 A region were also cast (Young et al. 1998).

The ESA/NASA Solar and Heliospheric Observatory (SOHO) has produced a wealth of spectral data with the CDS, SUMER, and UVCS instruments. The CDS covers a wide wavelength range (150-780 ̊) with nine channels, distributed between a normal incidence (NIS) and a grazing incidence (GIS) spectrometer. Data from CDS as described in Del Zanna (1999) were used for the benchmark, adopting the radiometric calibration of Del Zanna et al. (2001).

\subsection{Coronal forbidden lines}

Observations of coronal forbidden lines within the lower configurations are very important because differences in level energies can be measured with high accuracy $\left(\simeq 1 \mathrm{~cm}^{-1}\right)$. In turn, these level separations affect all the energies of the levels that mainly decay to these lower states. The visible coronal forbidden lines have been observed during total solar eclipses since 1869 , but the only study useful for the benchmark is the one by Jefferies et al. (1971).

The Skylab ATM NRL S082B spectrograph recorded a large number of coronal forbidden lines in the UV. Sandlin et al. (1977) provided an extensive and accurate list of calibrated line intensities and wavelengths in the 970-2650 A range. They estimated the relative intensities to be accurate within $30 \%$ in the 1210-1930 $\AA$ range and 50\% above $1930 \AA$, given the large scattered continuum. Wavelength measurements have an accuracy of $\simeq 0.01 \AA$. More lines were presented by Sandlin \& Tousey (1979). Feldman \& Doschek (1977) also provided a list of forbidden lines in the 1170-2650 $\AA$ range. Several
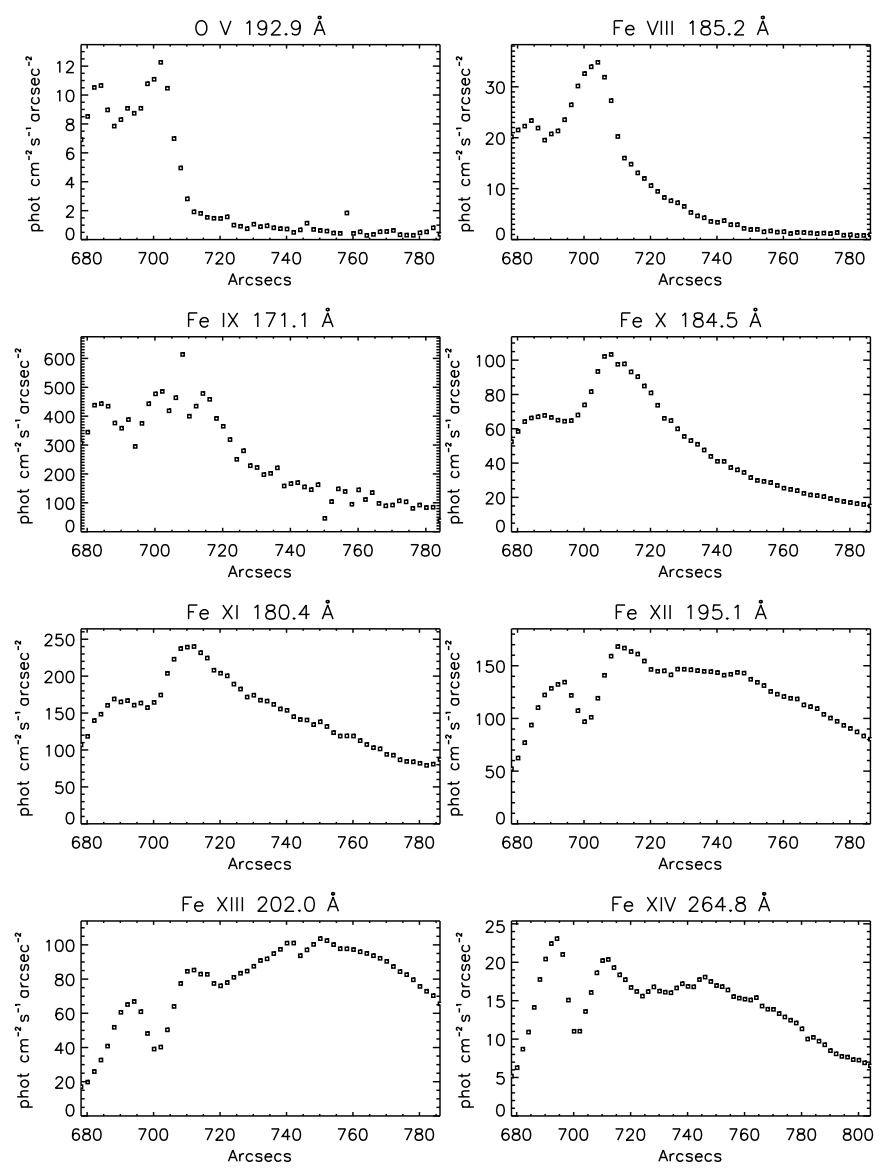

Fig. 1. Intensities in a selection of Hinode/EIS lines as they vary across the solar limb (arcsecs from solar centre along the N-S direction).

coronal forbidden lines have also been observed with the SOHO SUMER instrument in the in the 500-1600 $\AA$ range (see, e.g., Feldman et al. 1997). Many of them still await firm identifications.

\subsection{Hinode/EIS}

The entire Hinode/EIS database was searched for a suitable observation. An ideal spectrum was not found, however a few Hinode/EIS spectra were found to be useful for the benchmark. Only results from a single long-exposure (90 s) observation on March 11, 2007 are presented here. To avoid contamination from low-temperature lines, it is fundamental to observe off-limb. To limit contamination from high-temperature lines, a region of quiet Sun was selected. The $1^{\prime \prime}$ slit was moved to raster a region in the NW quadrant. The full spectral range (SW: 166-212 $\AA$; LW: 245-291 A) was telemetered to the ground.

A complex data processing, which included various geometrical corrections and a wavelength calibration procedure was applied to the data, as described in detail in Del Zanna (2009b). The main correction was for the slant in the SW and LW spectra, to ensure that different spectral regions were co-spatial (see Del Zanna \& Ishikawa 2009).

A few exposures were averaged to obtain a series of good spectra along the slit, crossing the solar limb. More than 200 lines were fitted with Gaussian profiles using the $c$ fit package (Haugan 1997) and their morphology examined in detail, one by one, to search for possible Fe XI lines. Figure 1 shows the intensities in a few selected lines as they vary across the 

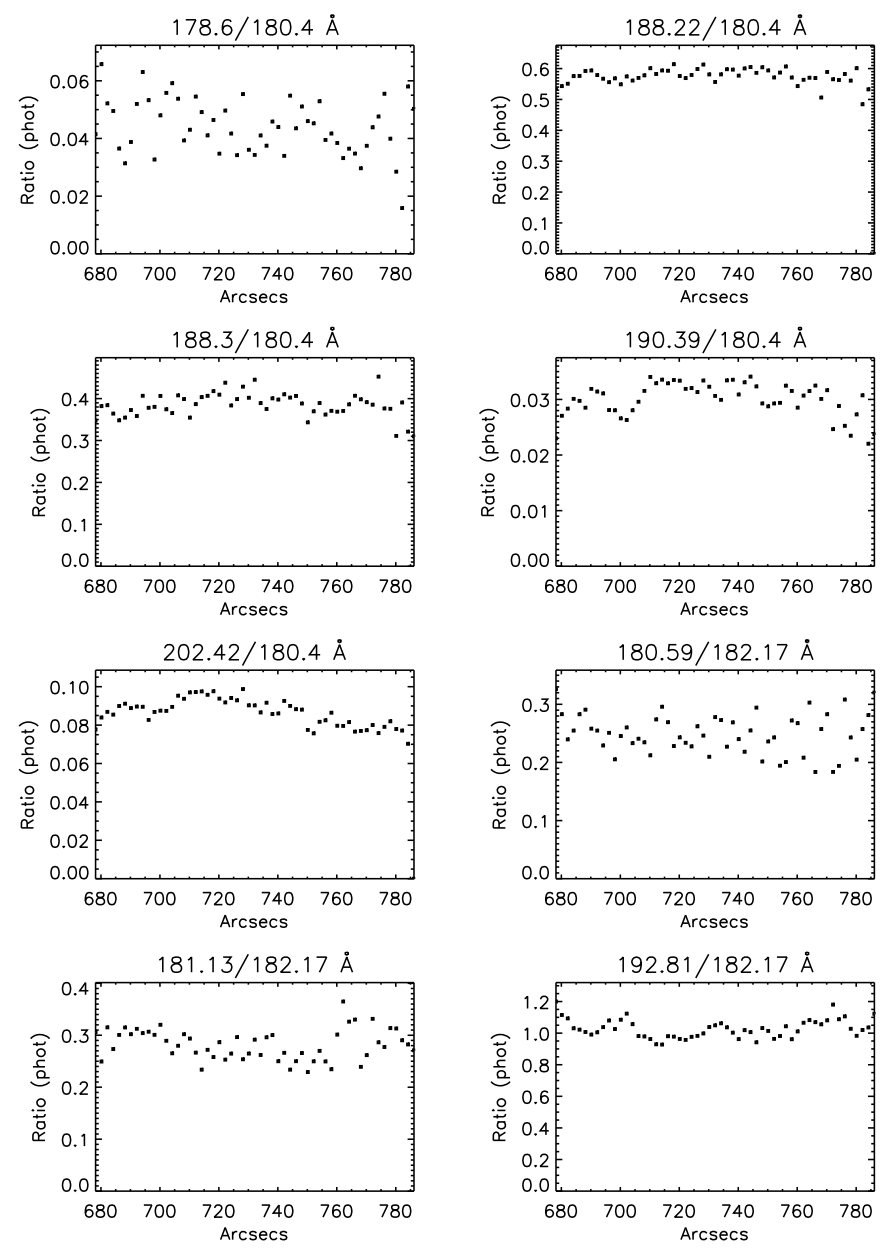

Fig. 2. Ratios of intensities in a selection of Hinode/EIS Fe XI lines as they vary across the solar limb.

solar limb. Notice the strong limb-brightening at $705^{\prime \prime}$ in cooler lines (cf. O V and Fe VIII), and in general the different morphology in lines formed at different temperatures. Figure 2 shows a selection of the Fe XI line ratios that are predicted to be fairly constant with density based on the present Fe XI model. These types of plots were used to assess the level of blending and the line identifications.

Furthermore, a region between 725 and 730" (see Fig. 1) was selected and an averaged spectrum obtained. This region is close enough to the limb so as to have a good signal in most lines, but far enough to limit blending with cooler lines formed at transition region temperatures such as those from $\mathrm{OV}$ and $\mathrm{Fe}$ VIII. A sample of spectral windows from this spectrum is provided in Fig. 3. The spectrum contained 343 lines, many of which still remain unidentified. Off-limb spectra of the quiet Sun are excellent for the benchmark, because they are nearly isodensity and isothermal (see below).

\section{Benchmark method}

The method, fully described in Paper I, is very simple in principle. A "benchmark" structure calculation is used to calculate the radiative data using the SUPERSTRUCTURE program (SS, see Eissner et al. 1974) and AUTOSTRUCTURE (Badnell 1997). $R$-matrix scattering calculations were performed to obtain approximate values for the excitations by electron impact. A model ion is built by including all the spectroscopically important configurations and all excitations/de-excitations between all the fine-structure levels. As outlined in Paper I, this is particularly important for complex ions with many metastable levels such as Fe XI.

The stationary level populations are then solved, obtaining $N_{j}\left(N_{\mathrm{e}}, T_{\mathrm{e}}\right)$, the population of level $j$ relative to the total $N\left(X^{+r}\right)$ number density of the ion $X^{+r}$, as a function of the electron temperature and density. The line intensities, $I_{\mathrm{th}}$, proportional to $N_{j} A_{j i}$, are then directly obtained by knowing $A_{j i}$, the spontaneous transition probability from the upper level $j$ to the lower level $i$. The identifications of the brightest lines are then considered, by comparing the "emissivity ratios"

$$
F_{j i}=\frac{I_{\mathrm{ob}} N_{\mathrm{e}} C}{N_{j}\left(N_{\mathrm{e}}, T_{0}\right) A_{j i}}
$$

calculated at a fixed temperature $T_{0}$ and plotted as a function of the electron density $N_{\mathrm{e}}$. $I_{\mathrm{ob}}$ is the observed intensity in the line. The scaling constant $C$ is chosen so the curves are close to unity. If there is agreement between theory and observations, all the $F_{j i}$ curves will either overlap or cross at one density value for isodensity and isothermal plasmas. Similar emissivity ratios as a function of temperature are obtained by fixing the electron density instead.

This approach is the same as analysing all the possible combinations of line ratios, as is commonly done in the literature. Departures from the isodensity or isothermal case can be important for some lines, but are normally second-order effects, compared to the uncertainties in the line identifications, line blending, instrument calibration, and in the atomic data. For Fe XI, the $F_{j i}$ curves have been calculated, unless otherwise stated, at $\log T_{0}[\mathrm{~K}]=6.11$, the approximate temperature of peak emission in ionization equilibrium (Dere et al. 2009).

The energy of the levels are then semi-empirically adjusted using the identified transitions. The semi-empirical term energy correction (TEC) procedure (see, e.g. Zeippen et al. 1977; Nussbaumer \& Storey 1978) is adopted, to obtain empiricallyadjusted fine-structure energies, $E_{\mathrm{SS}}$. For uncertain levels, the same corrections applied to other levels having the same parent term are used at first. The adjusted energies $E_{\mathrm{SS}}$ are then compared to the observed energies $E_{\mathrm{obs}}$, which are directly derived from the observed wavelengths $\lambda_{\text {obs }}$. Ground-based measurements have been converted to vacuum wavelengths using the standard formula for the refractive index of air given by Edlén (1966).

The method was applied iteratively many times, before a suitable and consistent set of energies and line intensities was obtained. At the end of the iterative procedure, a set of experimental energies $E_{\text {exp }}$ is provided, from which experimental wavelengths $\lambda_{\exp }$ are obtained. Notice that occasionally, the $\lambda_{\text {exp }}$ values can be slightly different from the observed ones. The final results from the benchmark structure with and without TEC are presented in Del Zanna et al. (2010), along with the final scattering calculation. The experimental energies $E_{\exp }$ were then used to calculate the $A$-values of all the E1, E2, M1, and M2 transitions in intermediate coupling with AUTOSTRUCTURE using the same target used for the scattering calculation.

\section{Summary of results}

The discussion is focused on the three main $n=3$ spectroscopic configurations in Fe XI, $3 s^{2} 3 p^{4}, 3 s 3 p^{5}, 3 s^{2} 3 p^{3} 3 d$, which produce 47 fine-structure levels, and all the brightest lines for this ion. Additional levels from $3 \mathrm{~s} 3 \mathrm{p}^{4} 3 \mathrm{~d}$ are also identified and 
Table 1. Level energies for Fe XI.

\begin{tabular}{|c|c|c|c|c|c|}
\hline$i$ & Conf. & $\mathrm{T}$ & $E_{\exp }$ & $E_{\mathrm{th}}$ & $E_{\mathrm{NIST}}$ \\
\hline 1 & $3 s^{2} 3 p^{4}$ & ${ }^{3} \mathrm{P}_{2}$ & 0 & 0 & $0(0)$ \\
\hline 2 & $3 s^{2} 3 p^{4}$ & ${ }^{3} \mathrm{P}_{1}$ & 12667.8 & $12587(81)$ & $12667.9(0)$ \\
\hline 3 & $3 s^{2} 3 p^{4}$ & ${ }^{3} \mathrm{P}_{0}$ & 14306 & $14245(61)$ & $14312(-6)$ \\
\hline 4 & $3 s^{2} 3 p^{4}$ & $\begin{array}{l}1 \\
{ }^{1} \mathrm{D}_{2}\end{array}$ & 37743 & $39504(-1761)$ & $37743.6(-1)$ \\
\hline 5 & $3 s^{2} 3 p^{4}$ & ${ }^{1} \mathrm{~S}_{0}$ & 80831 & $80190(641)$ & 80814.7 (16) \\
\hline 6 & $3 s 3 p^{5}$ & ${ }^{3} \mathrm{P}_{2}$ & 283451 & $279487(4064)$ & $283458(-7)$ \\
\hline 7 & $3 \mathrm{~s} 3 \mathrm{p}^{5}$ & ${ }^{3} \mathrm{P}_{1}$ & 293458 & $289478(3980)$ & $293458(0)$ \\
\hline 8 & $3 \mathrm{~s} 3 \mathrm{p}^{5}$ & ${ }^{3} \mathrm{P}_{0}$ & 299463 & 295416 (4047) & $299463(0)$ \\
\hline 9 & $3 \mathrm{~s} 3 \mathrm{p}^{5}$ & ${ }^{1} \mathrm{P}_{1}$ & 361446 & $362421(-675)$ & $361442(4)$ \\
\hline 10 & $3 s^{2} 3 p^{3} 3 d$ & ${ }^{5} \mathrm{D}_{0}$ & 387444 & 386437 (1507) & - \\
\hline 11 & $3 s^{2} 3 p^{3} 3 d$ & ${ }^{5} \mathrm{D}_{1}$ & 387426 & 386478 (1448) & - \\
\hline 12 & $3 s^{2} 3 p^{3} 3 d$ & ${ }^{5} \mathrm{D}_{2}$ & 387440 & 386484 (1356) & - \\
\hline 13 & $3 s^{2} 3 p^{3} 3 d$ & ${ }^{5} \mathrm{D}_{3}$ & 388468 & 387449 (1219) & - \\
\hline 14 & $3 s^{2} 3 p^{3} 3 d$ & ${ }^{5} \mathrm{D}_{4}$ & 389427 & 388480 (1047) & - \\
\hline 15 & $3 s^{2} 3 p^{3} 3 d$ & ${ }^{3} \mathrm{D}_{2}$ & 412456 & $413427(-471)$ & - \\
\hline 16 & $3 s^{2} 3 p^{3} 3 d$ & ${ }^{3} \mathrm{D}_{3}$ & 415426 & $415458(-532)$ & - \\
\hline 17 & $3 s^{2} 3 p^{3} 3 d$ & ${ }^{3} \mathrm{D}_{1}$ & 417449 & $417429(-480)$ & - \\
\hline 18 & $3 s^{2} 3 p^{3} 3 d$ & ${ }^{3} \mathrm{~F}_{2}$ & 422444 & $423471(-1027)$ & - \\
\hline 19 & $3 s^{2} 3 p^{3} 3 d$ & ${ }^{1} \mathrm{~S}_{0}$ & - & 426404 & - \\
\hline 20 & $3 s^{2} 3 p^{3} 3 d$ & ${ }^{3} \mathrm{~F}_{3}$ & 426422 & $427435(-1413)$ & - \\
\hline 21 & $3 s^{2} 3 p^{3} 3 d$ & ${ }^{3} \mathrm{~F}_{4}$ & 430422 & $432467(-1745)$ & - \\
\hline 22 & $3 s^{2} 3 p^{3} 3 d$ & ${ }^{3} \mathrm{G}_{3}$ & - & 452421 & - \\
\hline 23 & $3 s^{2} 3 p^{3} 3 d$ & ${ }^{3} \mathrm{G}_{4}$ & 450411 & $454413(-3902)$ & - \\
\hline 24 & $3 s^{2} 3 p^{3} 3 d$ & ${ }^{3} \mathrm{G}_{5}$ & 452416 & $456442(-4226)$ & - \\
\hline 25 & $3 s^{2} 3 p^{3} 3 d$ & ${ }^{1} \mathrm{G}_{4}$ & 459418 & $463479(-4561)$ & - \\
\hline 26 & $3 s^{2} 3 p^{3} 3 d$ & ${ }^{1} \mathrm{D}_{2}$ & - & 468495 & - \\
\hline 27 & $3 s^{2} 3 p^{3} 3 d$ & ${ }^{3} \mathrm{D}_{1}$ & - & 484412 & - \\
\hline 28 & $3 s^{2} 3 p^{3} 3 d$ & ${ }^{3} \mathrm{P}_{0}$ & - & 484477 & - \\
\hline 29 & $3 s^{2} 3 p^{3} 3 d$ & ${ }^{3} \mathrm{P}_{1}$ & 484430 & $486400(-2070)$ & - \\
\hline 30 & $3 s^{2} 3 p^{3} 3 d$ & ${ }^{3} \mathrm{~F}_{3}$ & 485439 & $488474(-3435)$ & - \\
\hline 31 & $3 s^{2} 3 p^{3} 3 d$ & ${ }^{3} \mathrm{~F}_{2}$ & - & 489460 & - \\
\hline 32 & $3 s^{2} 3 p^{3} 3 d$ & ${ }^{3} \mathrm{~F}_{4}$ & 486413 & $490469(-3656)$ & - \\
\hline 33 & $3 s^{2} 3 p^{3} 3 d$ & ${ }^{3} \mathrm{D}_{2}$ & 489478 & $492480(-3002)$ & - \\
\hline 34 & $3 s^{2} 3 p^{3} 3 d$ & ${ }^{3} \mathrm{P}_{2}$ & 494413 & $496444(-2531)$ & $496490(-2077)$ \\
\hline 35 & $3 s^{2} 3 p^{3} 3 d$ & ${ }^{3} \mathrm{D}_{3}$ & 497435 & $500455(-3320)$ & - \\
\hline 36 & $3 s^{2} 3 p^{3} 3 d$ & ${ }^{1} \mathrm{~F}_{3}$ & 525460 & $530447(-5087)$ & - \\
\hline 37 & $3 s^{2} 3 p^{3} 3 d$ & ${ }^{3} \mathrm{P}_{1}$ & 531470 & $536411(-5741)$ & $526480(4590)$ \\
\hline 38 & $3 s^{2} 3 p^{3} 3 d$ & ${ }^{3} \mathrm{P}_{2}$ & 531404 & $537400(-5696)$ & $531490(14)$ \\
\hline 39 & $3 s^{2} 3 p^{3} 3 d$ & ${ }^{3} \mathrm{~S}_{1}$ & 533445 & $540491(-6746)$ & $533450(-5)$ \\
\hline 40 & $3 s^{2} 3 p^{3} 3 d$ & ${ }^{3} \mathrm{P}_{0}$ & 541477 & $547499(-5822)$ & $541420(57)$ \\
\hline 41 & $3 s^{2} 3 p^{3} 3 d$ & ${ }^{3} \mathrm{P}_{1}$ & 541424 & $547432(-6308)$ & 541490 \\
\hline 42 & $3 s^{2} 3 p^{3} 3 d$ & ${ }^{3} \mathrm{D}_{3}$ & 554421 & $561499(-7378)$ & $554400(21)$ \\
\hline 43 & $3 s^{2} 3 p^{3} 3 d$ & ${ }^{3} \mathrm{D}_{2}$ & 561415 & $569408(-7693)$ & 561410 \\
\hline 44 & $3 s^{2} 3 p^{3} 3 d$ & ${ }^{3} \mathrm{D}_{1}$ & 566496 & $573492(-7596)$ & $566480(16)$ \\
\hline 45 & $3 s^{2} 3 p^{3} 3 d$ & ${ }^{1} \mathrm{D}_{2}$ & 578490 & $587476(-8886)$ & $578460(30)$ \\
\hline 46 & $3 s^{2} 3 p^{3} 3 d$ & ${ }^{1} \mathrm{~F}_{3}$ & 594447 & $603420(-9673)$ & $594430(17)$ \\
\hline 47 & $3 s^{2} 3 p^{3} 3 d$ & ${ }^{1} \mathrm{P}_{1}$ & 623401 & $631499(-7998)$ & $623480(21)$ \\
\hline 54 & $3 \mathrm{~s} 3 \mathrm{p}^{4} 3 \mathrm{~d}$ & ${ }^{5} \mathrm{~F}_{5}$ & 695472 & $696449(-577)$ & - \\
\hline 67 & $3 \mathrm{~s} 3 \mathrm{p}^{4} 3 \mathrm{~d}$ & ${ }^{3} \mathrm{~F}_{4}$ & 739460 & $741445(-2185)$ & - \\
\hline 79 & $3 \mathrm{~s} 3 \mathrm{p}^{4} 3 \mathrm{~d}$ & ${ }^{3} \mathrm{D}_{3}$ & 805492 & $807435(-1743)$ & - \\
\hline 103 & $3 \mathrm{~s} 3 \mathrm{p}^{4} 3 \mathrm{~d}$ & ${ }^{3} \mathrm{D}_{3}$ & 875416 & $877472(-1656)$ & - \\
\hline
\end{tabular}

Notes. Columns indicate the indices, the configuration, the dominant $L S J$, the experimental level energies $E_{\exp }\left(\mathrm{cm}^{-1}\right)$, those obtained from the scattering calculation $E_{\mathrm{th}}$, and those from NIST. Values in parentheses indicate differences with the experimental energies. Only a selection of observed levels from the lower configurations, producing the EUV lines discussed here, are presented.

briefly discussed. Table 1 lists the energies and identifications of the lowest 47 levels, together with a few from the $3 \mathrm{~s} 3 \mathrm{p}^{4} 3 \mathrm{~d}$ configuration. The $L S$ notation is ambiguous for many mixed levels. The experimental level energies $E_{\exp }\left(\mathrm{cm}^{-1}\right)$, together with those obtained from the scattering calculation $E_{\text {th }}$, and those from the National Institute of Standards and Technology (NIST) version 3 database $^{1}$ are displayed. Most levels are now finally firmly established. The Fe XI energies presented here turn out to be very close to the ab-initio values obtained by Ishikawa \& Vilkas (2008) using relativistic multi-reference many-body perturbation theory calculations, as shown in Del Zanna et al. (2010).

Table 2 lists the brightest lines (in decreasing order of intensity), grouped in three wavelength ranges, EUV, UV, and $\mathrm{UV} /$ visible. The wavelengths $\lambda_{\exp }$ corresponding to the experimental energies $E_{\text {exp }}$ are shown, with $g f$ and $A$ values. The wavelengths $\lambda_{\text {th }}$ obtained from the energies of the scattering calculation, and those from the NIST energies $\lambda_{\text {NIST }}$ are also shown. Notice the large differences between $\lambda_{\exp }$ and $\lambda_{\text {th }}$, which are however typical. Also, for many cases NIST wavelengths are close to $\lambda_{\text {exp }}$, although they are often significantly different, when high-resolution observations such as those from Hinode/EIS are considered.

It is very satisfactory to see that all the brightest transitions have been identified finally. Intensities were calculated at low $\left(10^{8} \mathrm{~cm}^{-3}\right)$ and high $\left(10^{12} \mathrm{~cm}^{-3}\right)$ densities, typical of the quiet-Sun off-limb corona and of laboratory spectra. Notice the striking difference in the relative intensities of some lines, at different densities. The benchmark and identification using different sources took this into account.

A summary of the proposed identifications is given in Table 3 . It lists the observed wavelengths $\lambda_{\text {obs }}$ that have been assessed to be best and used to obtain the experimental energies $E_{\text {exp }}$. Some of the lines are self-blended or blend with transitions from other ions. The most common ones are indicated in the table. However, blending depends on the instrument resolution and on the plasma source. The table also indicates some of the most relevant previous identifications whether consistent or not with the present ones. The reader should keep in mind that it is virtually impossible to provide details for all the line identifications proposed, discussed, or rejected in the literature.

\section{Energy levels and line identifications}

An in-depth discussion of the main spectral identifications for each of the levels is essential. In what follows, level energies are given in $\mathrm{cm}^{-1}$.

\subsection{Ground configuration $3 s^{2} 3 p^{4}$ and forbidden transitions}

The splitting between the ${ }^{3} \mathrm{P}_{2,1}^{\mathrm{e}}$ levels is accurately measured by the 1-2 transition observed by Jefferies et al. (1971) at $7891.8 \AA$ (7894.0 $\AA$ in vacuum, which provides 12 667.8). This is in good agreement with the wavelength differences between decays from the same upper level to levels 1,2. For example, the decays from level 37, observed at 188.216 and $192.813 \AA$, provide an energy of 12 667.2. The energy of level 3 is obtained from the wavelengths of the 3-44 and 2-44 lines observed at 181.131 and $180.595 \AA$, and the energy of level 2 . The energy of level 4 , 37743 , is from the strong 1-4 transition observed by Sandlin et al. (1977) at $2648.71 \AA$ (air). The wavelength of the 2-4 line observed by Jefferies et al. (1971) at $3986.8 \AA$ (air) provides an energy of 37743.6 , in good agreement.

The energy of level 5 is from the bright 2-5 line observed by Sandlin et al. (1977) at $1467.06 \AA$. Jordan (1971) used the 1970 eclipse observations described by Gabriel et al. (1971) to

\footnotetext{
1 http://physics.nist.gov
} 
G. Del Zanna: Fe XI benchmark

Table 2. List of the brightest Fe XI lines, from the EUV to the visible.

\begin{tabular}{|c|c|c|c|c|c|c|c|c|c|}
\hline$i-j$ & Levels & $\begin{array}{c}\text { Int } \\
1 \times 10^{8} \\
\end{array}$ & $\begin{array}{r}\text { Int } \\
1 \times 10^{12} \\
\end{array}$ & $g f$ & $\begin{array}{r}A_{j i} \\
\left(\mathrm{~s}^{-1}\right) \\
\end{array}$ & $\begin{array}{c}A_{j i} \\
\text { NIST } \\
\end{array}$ & $\begin{array}{l}\lambda_{\exp } \\
(\AA)\end{array}$ & $\begin{array}{l}\lambda_{\mathrm{th}} \\
(\AA)\end{array}$ & $\begin{array}{c}\lambda_{\text {NIST }} \\
(\AA) \\
\end{array}$ \\
\hline $1-42$ & $3 p^{4}{ }^{3} P_{2}-3 d^{3} D_{3}$ & 1.0 & 0.43 & 4.78 & $1.4 \times 10^{11}$ & - & 180.401 & 178.04 & 180.408 \\
\hline $1-38$ & $3 p^{4}{ }^{3} P_{2}-3 d{ }^{3} P_{2}$ & 0.50 & 0.25 & 2.61 & $9.7 \times 10^{10}$ & $1.1 \times 10^{11}$ & 188.216 & 186.22 & 188.221 \\
\hline $1-37$ & $3 p^{4}{ }^{3} P_{2}-3 d^{3} P_{1}$ & 0.30 & 0.15 & 1.52 & $9.5 \times 10^{10}$ & - & 188.299 & 186.27 & 189.941 \\
\hline $2-43$ & $3 p^{4}{ }^{3} P_{1}-3 d^{3} D_{2}$ & 0.12 & 0.19 & 2.63 & $1.0 \times 10^{11}$ & - & 182.167 & 179.62 & 182.169 \\
\hline $2-38$ & $3 \mathrm{p}^{4}{ }^{3} \mathrm{P}_{1}-3 \mathrm{~d}^{3} \mathrm{P}_{2}$ & 0.10 & $5.2 \times 10^{-2}$ & 0.57 & $2.0 \times 10^{10}$ & $2.2 \times 10^{10}$ & 192.813 & 190.70 & 192.819 \\
\hline $1-14$ & $3 p^{4}{ }^{3} \mathrm{P}_{2}-3 d^{5} \mathrm{D}_{4}$ & $8.3 \times 10^{-2}$ & $1.3 \times 10^{-4}$ & - & 35. & - & 256.919 & 257.65 & - \\
\hline $1-16$ & $3 p^{4}{ }^{3} P_{2}-3 d^{3} D_{3}$ & $5.8 \times 10^{-2}$ & $4.9 \times 10^{-2}$ & $1.6 \times 10^{-4}$ & $2.6 \times 10^{6}$ & - & 240.717 & 240.41 & - \\
\hline $3-44$ & $3 \mathrm{p}^{4}{ }^{3} \mathrm{P}_{0}-3 \mathrm{~d}^{3} \mathrm{D}_{1}$ & $3.9 \times 10^{-2}$ & $7.8 \times 10^{-2}$ & 1.18 & $7.9 \times 10^{10}$ & - & 181.130 & 178.65 & 181.137 \\
\hline $4-37$ & $3 \mathrm{p}^{4}{ }^{1} \mathrm{D}_{2}-3 \mathrm{~d}^{3} \mathrm{P}_{1}$ & $4.2 \times 10^{-2}$ & $2.1 \times 10^{-2}$ & 0.25 & $1.4 \times 10^{10}$ & - & 202.705 & 201.06 & 204.609 \\
\hline $1-34$ & $3 p^{4}{ }^{3} P_{2}-3 d^{3} P_{2}$ & $3.9 \times 10^{-2}$ & $2.7 \times 10^{-2}$ & 0.15 & $5.0 \times 10^{9}$ & $3.6 \times 10^{9}$ & 202.424 & 201.41 & 201.576 \\
\hline $1-43$ & $3 p^{4}{ }^{3} P_{2}-3 d^{3} D_{2}$ & $3.2 \times 10^{-2}$ & $5.1 \times 10^{-2}$ & 0.68 & $2.8 \times 10^{10}$ & - & 178.058 & 175.64 & 178.060 \\
\hline $1-13$ & $3 p^{4}{ }^{3} P_{2}-3 d^{5} D_{3}$ & $4.1 \times 10^{-2}$ & $4.6 \times 10^{-2}$ & $1.5 \times 10^{-3}$ & $2.1 \times 10^{7}$ & - & 257.554 & 258.37 & - \\
\hline $2-44$ & $3 p^{4}{ }^{3} P_{1}-3 d^{3} D_{1}$ & $2.8 \times 10^{-2}$ & $5.6 \times 10^{-2}$ & 0.83 & $5.6 \times 10^{10}$ & - & 180.594 & 178.12 & 180.599 \\
\hline $4-39$ & $3 \mathrm{p}^{4}{ }^{1} \mathrm{D}_{2}-3 \mathrm{~d}^{3} \mathrm{~S}_{1}$ & $2.3 \times 10^{-2}$ & $6.6 \times 10^{-2}$ & 0.91 & $4.9 \times 10^{10}$ & - & 201.734 & 199.73 & 201.732 \\
\hline $4-21$ & $3 \mathrm{p}^{4}{ }^{1} \mathrm{D}_{2}-3 \mathrm{~d}^{3} \mathrm{~F}_{4}$ & $2.6 \times 10^{-2}$ & $1.5 \times 10^{-4}$ & - & 57. & - & 254.596 & 254.66 & - \\
\hline $1-12$ & $3 \mathrm{p}^{4}{ }^{3} \mathrm{P}_{2}-3 \mathrm{~d}^{5} \mathrm{D}_{2}$ & $2.5 \times 10^{-2}$ & $2.6 \times 10^{-2}$ & $3.2 \times 10^{-3}$ & $6.4 \times 10^{7}$ & - & 257.772 & 258.64 & - \\
\hline $4-41$ & $3 \mathrm{p}^{4}{ }^{1} \mathrm{D}_{2}-3 \mathrm{~d}^{3} \mathrm{P}_{1}$ & $1.8 \times 10^{-2}$ & $5.3 \times 10^{-2}$ & 0.77 & $4.3 \times 10^{10}$ & - & 198.538 & 196.77 & 198.552 \\
\hline $1-15$ & $3 p^{4}{ }^{3} P_{2}-3 d^{3} D_{2}$ & $2.1 \times 10^{-2}$ & $2.2 \times 10^{-2}$ & $1.6 \times 10^{-3}$ & $3.7 \times 10^{7}$ & - & 242.215 & 241.91 & - \\
\hline $1-29$ & $3 p^{4}{ }^{3} P_{2}-3 d^{3} P_{1}$ & $1.7 \times 10^{-2}$ & $1.1 \times 10^{-2}$ & $5.2 \times 10^{-2}$ & $2.7 \times 10^{9}$ & - & 206.258 & 205.38 & - \\
\hline $1-35$ & $3 p^{4}{ }^{3} P_{2}-3 d^{3} D_{3}$ & $1.6 \times 10^{-2}$ & $2.2 \times 10^{-2}$ & $2.1 \times 10^{-2}$ & $5.0 \times 10^{8}$ & - & 201.112 & 199.81 & - \\
\hline $2-41$ & $3 p^{4}{ }^{3} P_{1}-3 d^{3} P_{1}$ & $1.5 \times 10^{-2}$ & $4.5 \times 10^{-2}$ & 0.59 & $3.6 \times 10^{10}$ & - & 189.123 & 186.88 & 189.135 \\
\hline $1-18$ & $3 p^{4}{ }^{3} P_{2}-3 d^{3} F_{2}$ & $1.8 \times 10^{-2}$ & $2.2 \times 10^{-2}$ & $8.2 \times 10^{-4}$ & $2.0 \times 10^{7}$ & - & 236.494 & 235.87 & - \\
\hline $4-16$ & $3 \mathrm{p}^{4}{ }^{1} \mathrm{D}_{2}-3 \mathrm{~d}^{3} \mathrm{D}_{3}$ & $1.9 \times 10^{-2}$ & $1.6 \times 10^{-2}$ & $6.1 \times 10^{-5}$ & $8.3 \times 10^{5}$ & - & 264.772 & 265.63 & - \\
\hline $2-39$ & $3 p^{4}{ }^{3} P_{1}-3 d^{3} S_{1}$ & $1.3 \times 10^{-2}$ & $3.7 \times 10^{-2}$ & 0.47 & $2.8 \times 10^{10}$ & - & 192.021 & 189.55 & 192.019 \\
\hline $3-41$ & $3 \mathrm{p}^{4}{ }^{3} \mathrm{P}_{0}-3 \mathrm{~d}^{3} \mathrm{P}_{1}$ & $1.3 \times 10^{-2}$ & $3.7 \times 10^{-2}$ & 0.49 & $3.0 \times 10^{10}$ & - & 189.711 & 187.47 & 189.725 \\
\hline $1-36$ & $3 p^{4}{ }^{3} P_{2}-3 d^{1} F_{3}$ & $1.2 \times 10^{-2}$ & $1.9 \times 10^{-2}$ & $3.3 \times 10^{-2}$ & $8.7 \times 10^{8}$ & - & 190.382 & 188.56 & - \\
\hline $3-37$ & $3 p^{4}{ }^{3} P_{0}-3 d^{3} P_{1}$ & $1.2 \times 10^{-2}$ & $5.9 \times 10^{-3}$ & $6.4 \times 10^{-2}$ & $3.8 \times 10^{9}$ & - & 193.512 & 191.36 & 195.248 \\
\hline $4-20$ & $3 \mathrm{p}^{4}{ }^{1} \mathrm{D}_{2}-3 \mathrm{~d}^{3} \mathrm{~F}_{3}$ & $1.5 \times 10^{-2}$ & $2.1 \times 10^{-2}$ & $7.4 \times 10^{-4}$ & $1.1 \times 10^{7}$ & - & 257.547 & 257.77 & - \\
\hline $1-20$ & $3 p^{4}{ }^{3} P_{2}-3 d^{3} F_{3}$ & $1.3 \times 10^{-2}$ & $1.8 \times 10^{-2}$ & $5.3 \times 10^{-4}$ & $9.1 \times 10^{6}$ & - & 234.730 & 233.96 & - \\
\hline $4-46$ & $3 \mathrm{p}^{4}{ }^{1} \mathrm{D}_{2}-3 \mathrm{~d}^{1} \mathrm{~F}_{3}$ & $9.2 \times 10^{-3}$ & 0.29 & 5.20 & $1.5 \times 10^{11}$ & $1.7 \times 10^{11}$ & 179.758 & 177.24 & 179.764 \\
\hline $2-33$ & $3 p^{4}{ }^{3} P_{1}-3 d^{3} D_{2}$ & $1.0 \times 10^{-2}$ & $1.4 \times 10^{-2}$ & $5.1 \times 10^{-2}$ & $1.5 \times 10^{9}$ & - & 209.771 & 208.42 & - \\
\hline $4-26$ & $3 \mathrm{p}^{4}{ }^{1} \mathrm{D}_{2}-3 \mathrm{~d}^{1} \mathrm{D}_{2}$ & $1.1 \times 10^{-2}$ & $2.1 \times 10^{-2}$ & $4.6 \times 10^{-2}$ & $1.1 \times 10^{9}$ & - & - & 233.09 & - \\
\hline $1-17$ & $3 p^{4}{ }^{3} P_{2}-3 d^{3} D_{1}$ & $9.9 \times 10^{-3}$ & $1.1 \times 10^{-2}$ & $5.0 \times 10^{-4}$ & $1.9 \times 10^{7}$ & - & 239.780 & 239.48 & - \\
\hline $2-34$ & $3 p^{4}{ }^{3} P_{1}-3 d^{3} P_{2}$ & $8.5 \times 10^{-3}$ & $5.8 \times 10^{-3}$ & $3.5 \times 10^{-2}$ & $1.1 \times 10^{9}$ & - & 207.751 & 206.65 & 206.859 \\
\hline $1-30$ & $3 p^{4}{ }^{3} P_{2}-3 d^{3} F_{3}$ & $8.1 \times 10^{-3}$ & $1.5 \times 10^{-2}$ & $8.1 \times 10^{-3}$ & $1.8 \times 10^{8}$ & - & 206.169 & 204.72 & - \\
\hline $4-22$ & $3 \mathrm{p}^{4}{ }^{1} \mathrm{D}_{2}-3 \mathrm{~d}^{3} \mathrm{G}_{3}$ & $9.4 \times 10^{-3}$ & $1.5 \times 10^{-2}$ & $3.4 \times 10^{-3}$ & $5.5 \times 10^{7}$ & - & - & 242.15 & - \\
\hline $4-38$ & $3 p^{4}{ }^{1} D_{2}-3 d^{3} P_{2}$ & $7.8 \times 10^{-3}$ & $3.9 \times 10^{-3}$ & $4.8 \times 10^{-2}$ & $1.5 \times 10^{9}$ & - & 202.609 & 201.00 & 202.615 \\
\hline $3-39$ & $3 p^{4}{ }^{3} P_{0}-3 d^{3} S_{1}$ & $7.2 \times 10^{-3}$ & $2.0 \times 10^{-2}$ & 0.25 & $1.5 \times 10^{10}$ & - & 192.627 & 190.15 & 192.627 \\
\hline $6-103$ & $3 p^{5}{ }^{3} P_{2}-3 d^{3} D_{3}$ & $5.3 \times 10^{-3}$ & $3.6 \times 10^{-3}$ & 4.13 & $1.4 \times 10^{11}$ & - & 168.929 & 167.32 & - \\
\hline $1-11$ & $3 p^{4}{ }^{3} P_{2}-3 d^{5} D_{1}$ & $8.0 \times 10^{-3}$ & $7.7 \times 10^{-3}$ & $1.7 \times 10^{-3}$ & $5.7 \times 10^{7}$ & - & 257.914 & 258.81 & - \\
\hline $4-45$ & $3 \mathrm{p}^{4}{ }^{1} \mathrm{D}_{2}-3 \mathrm{~d}^{1} \mathrm{D}_{2}$ & $5.6 \times 10^{-3}$ & 0.16 & 2.69 & $1.0 \times 10^{11}$ & $1.2 \times 10^{11}$ & 184.793 & 182.39 & 184.803 \\
\hline $3-27$ & $3 \mathrm{p}^{4}{ }^{3} \mathrm{P}_{0}-3 \mathrm{~d}^{3} \mathrm{D}_{1}$ & $6.3 \times 10^{-3}$ & $6.1 \times 10^{-3}$ & $2.0 \times 10^{-2}$ & $1.0 \times 10^{9}$ & - & - & 212.80 & - \\
\hline $1-22$ & $3 p^{4}{ }^{3} P_{2}-3 d^{3} G_{3}$ & $6.3 \times 10^{-3}$ & $1.0 \times 10^{-2}$ & $1.9 \times 10^{-3}$ & $3.7 \times 10^{7}$ & - & - & 221.02 & - \\
\hline $4-15$ & $3 \mathrm{p}^{4}{ }^{1} \mathrm{D}_{2}-3 \mathrm{~d}^{3} \mathrm{D}_{2}$ & $7.3 \times 10^{-3}$ & $7.4 \times 10^{-3}$ & $6.7 \times 10^{-4}$ & $1.3 \times 10^{7}$ & - & 266.586 & 267.46 & - \\
\hline $2-17$ & $3 p^{4}{ }^{3} P_{1}-3 d^{3} D_{1}$ & $6.4 \times 10^{-3}$ & $7.1 \times 10^{-3}$ & $3.4 \times 10^{-4}$ & $1.3 \times 10^{7}$ & - & 247.291 & 246.94 & - \\
\hline $4-31$ & $3 \mathrm{p}^{4}{ }^{1} \mathrm{D}_{2}-3 \mathrm{~d}^{3} \mathrm{~F}_{2}$ & $5.3 \times 10^{-3}$ & $9.8 \times 10^{-3}$ & $3.1 \times 10^{-3}$ & $8.5 \times 10^{7}$ & - & - & 222.26 & - \\
\hline $2-40$ & $3 \mathrm{p}^{4}{ }^{3} \mathrm{P}_{1}-3 \mathrm{~d}^{3} \mathrm{P}_{0}$ & $4.4 \times 10^{-3}$ & $5.4 \times 10^{-2}$ & 0.70 & $1.3 \times 10^{11}$ & $1.4 \times 10^{11}$ & 188.997 & 186.91 & 189.017 \\
\hline $1-39$ & $3 p^{4}{ }^{3} P_{2}-3 d^{3} S_{1}$ & $3.7 \times 10^{-3}$ & $1.0 \times 10^{-2}$ & 0.12 & $7.8 \times 10^{9}$ & - & 187.461 & 185.13 & 187.459 \\
\hline $21-79$ & $3 d^{3} F_{4}-3 d^{3} D_{3}$ & $5.3 \times 10^{-3}$ & $4.7 \times 10^{-3}$ & 0.55 & $7.3 \times 10^{9}$ & - & 266.759 & 266.70 & - \\
\hline $4-43$ & $3 p^{4}{ }^{1} D_{2}-3 d^{3} D_{2}$ & $3.2 \times 10^{-3}$ & $5.1 \times 10^{-3}$ & $7.7 \times 10^{-2}$ & $2.8 \times 10^{9}$ & - & 190.886 & 188.73 & 190.888 \\
\hline $2-19$ & $3 p^{4}{ }^{3} P_{1}-3 d^{1} S_{0}$ & $3.7 \times 10^{-3}$ & $5.1 \times 10^{-3}$ & $5.4 \times 10^{-4}$ & $6.2 \times 10^{7}$ & - & - & 241.71 & - \\
\hline $1-41$ & $3 p^{4}{ }^{3} P_{2}-3 d^{3} P_{1}$ & $2.3 \times 10^{-3}$ & $6.9 \times 10^{-3}$ & $8.6 \times 10^{-2}$ & $5.5 \times 10^{9}$ & - & 184.698 & 182.58 & 184.710 \\
\hline $5-41$ & $3 \mathrm{p}^{4}{ }^{1} \mathrm{~S}_{0}-3 \mathrm{~d}^{3} \mathrm{P}_{1}$ & $1.9 \times 10^{-3}$ & $5.7 \times 10^{-3}$ & $9.9 \times 10^{-2}$ & $4.6 \times 10^{9}$ & - & 217.111 & 213.88 & 217.120 \\
\hline $5-47$ & $3 \mathrm{p}^{4}{ }^{1} \mathrm{~S}_{0}-3 \mathrm{~d}^{1} \mathrm{P}_{1}$ & $1.4 \times 10^{-3}$ & $7.0 \times 10^{-2}$ & 1.88 & $1.2 \times 10^{11}$ & $1.4 \times 10^{11}$ & 184.410 & 181.52 & 184.412 \\
\hline $14-136$ & $3 d^{5} D_{4}-3 d^{2}{ }^{5} D_{4}$ & $8.7 \times 10^{-4}$ & $1.2 \times 10^{-2}$ & 5.38 & $1.3 \times 10^{11}$ & - & - & 174.42 & - \\
\hline $24-157$ & $3 d^{3} \mathrm{G}_{5}-3 \mathrm{~d}^{2}{ }^{3} \mathrm{H}_{6}$ & $8.3 \times 10^{-4}$ & $8.7 \times 10^{-3}$ & 4.13 & $6.2 \times 10^{10}$ & - & - & 187.71 & - \\
\hline $14-118$ & $3 d^{5} D_{4}-3 d^{2}{ }^{5} F_{5}$ & $7.7 \times 10^{-4}$ & $8.4 \times 10^{-3}$ & 2.36 & $3.7 \times 10^{10}$ & - & - & 195.67 & - \\
\hline 24-189 & $3 d^{3} G_{5}-3 d^{2}{ }^{3} G_{5}$ & $5.4 \times 10^{-4}$ & $8.1 \times 10^{-3}$ & 5.06 & $1.1 \times 10^{11}$ & - & - & 172.00 & - \\
\hline $2-45$ & $3 p^{4}{ }^{3} \mathrm{P}_{1}-3 \mathrm{~d}^{1} \mathrm{D}_{2}$ & $3.3 \times 10^{-4}$ & $9.4 \times 10^{-3}$ & 0.15 & $6.1 \times 10^{9}$ & $8.6 \times 10^{9}$ & 176.609 & 173.87 & 176.618 \\
\hline $1-6$ & $3 \mathrm{p}^{4}{ }^{3} \mathrm{P}_{2}-3 \mathrm{p}^{5}{ }^{3} \mathrm{P}_{2}$ & 0.20 & 0.12 & 0.22 & $2.4 \times 10^{9}$ & $1.7 \times 10^{9}$ & 352.670 & 357.79 & 352.662 \\
\hline $2-6$ & $3 p^{4}{ }^{3} \mathrm{P}_{1}-3 \mathrm{p}^{5}{ }^{3} \mathrm{P}_{2}$ & $6.2 \times 10^{-2}$ & $3.7 \times 10^{-2}$ & $7.6 \times 10^{-2}$ & $7.5 \times 10^{8}$ & $5.3 \times 10^{8}$ & 369.163 & 374.69 & 369.153 \\
\hline
\end{tabular}


Table 2. continued.

\begin{tabular}{|c|c|c|c|c|c|c|c|c|c|}
\hline$i-j$ & Levels & $\begin{array}{c}\text { Int } \\
1 \times 10^{8}\end{array}$ & $\begin{array}{r}\text { Int } \\
1 \times 10^{12}\end{array}$ & $g f$ & $A_{j i}\left(\mathrm{~s}^{-1}\right)$ & $\begin{array}{c}A_{j i}\left(\mathrm{~s}^{-1}\right) \\
\text { NIST }\end{array}$ & $\lambda_{\exp }(\AA)$ & $\lambda_{\mathrm{th}}(\AA)$ & $\lambda_{\mathrm{NIST}}(\AA)$ \\
\hline $1-7$ & $3 p^{4}{ }^{3} P_{2}-3 p^{5}{ }^{3} P_{1}$ & $4.9 \times 10^{-2}$ & $4.0 \times 10^{-2}$ & $7.9 \times 10^{-2}$ & $1.5 \times 10^{9}$ & $1.1 \times 10^{9}$ & 341.113 & 345.83 & 341.113 \\
\hline $16-67$ & $3 d^{3} D_{3}-3 d^{3} F_{4}$ & $2.9 \times 10^{-2}$ & $1.3 \times 10^{-2}$ & 0.25 & $2.0 \times 10^{9}$ & - & 308.991 & 307.42 & - \\
\hline $3-7$ & $3 p^{4}{ }^{3} \mathrm{P}_{0}-3 \mathrm{p}^{5}{ }^{3} \mathrm{P}_{1}$ & $3.3 \times 10^{-2}$ & $2.7 \times 10^{-2}$ & $5.9 \times 10^{-2}$ & $1.0 \times 10^{9}$ & $7.1 \times 10^{8}$ & 358.613 & 363.78 & 358.621 \\
\hline $4-9$ & $3 \mathrm{p}^{4}{ }^{1} \mathrm{D}_{2}-3 \mathrm{p}^{5}{ }^{1} \mathrm{P}_{1}$ & $2.5 \times 10^{-2}$ & $8.8 \times 10^{-2}$ & 0.37 & $8.7 \times 10^{9}$ & $7.5 \times 10^{9}$ & 308.544 & 309.57 & 308.548 \\
\hline $2-7$ & $3 p^{4}{ }^{3} P_{1}-3 p^{5}{ }^{3} P_{1}$ & $2.6 \times 10^{-2}$ & $2.1 \times 10^{-2}$ & $4.6 \times 10^{-2}$ & $8.1 \times 10^{8}$ & $5.2 \times 10^{8}$ & 356.519 & 361.58 & 356.519 \\
\hline $2-8$ & $3 p^{4}{ }^{3} \mathrm{P}_{1}-3 \mathrm{p}^{5}{ }^{3} \mathrm{P}_{0}$ & $1.0 \times 10^{-2}$ & $2.6 \times 10^{-2}$ & $6.1 \times 10^{-2}$ & $3.4 \times 10^{9}$ & $2.3 \times 10^{9}$ & 349.046 & 353.95 & 349.046 \\
\hline $14-54$ & $3 d^{5} D_{4}-3 d{ }^{5} F_{5}$ & $5.2 \times 10^{-3}$ & $1.1 \times 10^{-2}$ & 0.79 & $4.5 \times 10^{9}$ & - & 326.323 & 324.55 & - \\
\hline $4-6$ & $3 p^{4}{ }^{1} D_{2}-3 p^{5}{ }^{3} P_{2}$ & $6.1 \times 10^{-3}$ & $3.6 \times 10^{-3}$ & $8.9 \times 10^{-3}$ & $7.4 \times 10^{7}$ & $4.0 \times 10^{7}$ & 406.822 & 416.65 & 406.811 \\
\hline $2-5$ & $3 p^{4}{ }^{3} \mathrm{P}_{1}-3 \mathrm{p}^{4}{ }^{1} \mathrm{~S}_{0}$ & $4.1 \times 10^{-2}$ & $5.1 \times 10^{-3}$ & - & $9.8 \times 10^{2}$ & $9.8 \times 10^{2}$ & 1467.07 & 1480.36 & 1467.420 \\
\hline $6-21$ & $3 p^{5}{ }^{3} \mathrm{P}_{2}-3 d^{3} \mathrm{~F}_{4}$ & $1.4 \times 10^{-2}$ & $8.2 \times 10^{-5}$ & - & 30. & - & 680.406 & 654.97 & - \\
\hline $14-23$ & $3 d^{5} D_{4}-3 d^{3} G_{4}$ & $1.1 \times 10^{-2}$ & $2.3 \times 10^{-5}$ & - & 12. & - & 1639.77 & 1514.33 & - \\
\hline $14-25$ & $3 d^{5} D_{4}-3 d{ }^{1} G_{4}$ & $7.7 \times 10^{-3}$ & $6.5 \times 10^{-5}$ & - & 25 . & - & 1428.76 & 1321.72 & - \\
\hline $14-32$ & $3 d^{5} D_{4}-3 d^{3} F_{4}$ & $4.6 \times 10^{-3}$ & $3.5 \times 10^{-4}$ & - & $2.3 \times 10^{2}$ & - & 1028.95 & 981.61 & - \\
\hline $14-24$ & $3 d^{5} D_{4}-3 d^{3} G_{5}$ & $5.3 \times 10^{-3}$ & $6.2 \times 10^{-6}$ & - & 2.1 & - & 1582.55 & 1461.62 & - \\
\hline $13-25$ & $3 d^{5} D_{3}-3 d{ }^{1} G_{4}$ & $1.9 \times 10^{-3}$ & $1.6 \times 10^{-5}$ & - & 6.1 & - & 1409.44 & 1303.15 & - \\
\hline $13-23$ & $3 d^{5} D_{3}-3 d^{3} G_{4}$ & $1.2 \times 10^{-3}$ & $2.6 \times 10^{-6}$ & - & 1.3 & - & 1614.39 & 1490.00 & - \\
\hline $13-32$ & $3 d^{5} D_{3}-3 d{ }^{3} F_{4}$ & $8.7 \times 10^{-4}$ & $6.6 \times 10^{-5}$ & - & 44. & - & 1018.90 & 971.34 & - \\
\hline $6-14$ & $3 p^{5}{ }^{3} P_{2}-3 d{ }^{5} D_{4}$ & $7.1 \times 10^{-4}$ & $1.1 \times 10^{-6}$ & - & 0.30 & - & 946.29 & 920.56 & - \\
\hline $16-32$ & $3 d^{3} D_{3}-3 d^{3} F_{4}$ & $7.6 \times 10^{-4}$ & $5.7 \times 10^{-5}$ & - & 38. & - & 1408.71 & 1350.57 & - \\
\hline $13-24$ & $3 d^{5} D_{3}-3 d^{3} G_{5}$ & $1.3 \times 10^{-4}$ & $1.6 \times 10^{-7}$ & - & $5.3 \times 10^{-2}$ & - & 1558.90 & 1438.95 & - \\
\hline $1-5$ & $3 \mathrm{p}^{4}{ }^{3} \mathrm{P}_{2}-3 \mathrm{p}^{4}{ }^{1} \mathrm{~S}_{0}$ & $5.0 \times 10^{-5}$ & $6.3 \times 10^{-6}$ & - & 1.2 & 1.7 & 1237.15 & 1247.58 & 1237.399 \\
\hline $1-2$ & $3 p^{4}{ }^{3} \mathrm{P}_{2}-3 \mathrm{p}^{4}{ }^{3} \mathrm{P}_{1}$ & 0.85 & $1.4 \times 10^{-3}$ & - & 44. & 44. & 7894.03 & 7933.99 & 7893.968 \\
\hline $1-4$ & $3 \mathrm{p}^{4}{ }^{3} \mathrm{P}_{2}-3 \mathrm{p}^{4}{ }^{1} \mathrm{D}_{2}$ & 0.42 & $3.5 \times 10^{-3}$ & - & 84. & 92. & 2649.50 & 2532.67 & 2649.456 \\
\hline $2-4$ & $3 \mathrm{p}^{4}{ }^{3} \mathrm{P}_{1}-3 \mathrm{p}^{4}{ }^{1} \mathrm{D}_{2}$ & $4.3 \times 10^{-2}$ & $3.6 \times 10^{-4}$ & - & 8.5 & 9.5 & 3988.00 & 3720.24 & 3987.924 \\
\hline $14-21$ & $3 d^{5} D_{4}-3 d^{3} F_{4}$ & $1.3 \times 10^{-2}$ & $7.8 \times 10^{-5}$ & - & 29. & - & 2421.60 & 2270.15 & - \\
\hline $21-24$ & $3 d^{3} F_{4}-3 d^{3} G_{5}$ & $1.8 \times 10^{-2}$ & $2.1 \times 10^{-5}$ & - & 7.0 & - & 4567.46 & 4103.91 & - \\
\hline $21-23$ & $3 d^{3} F_{4}-3 d{ }^{3} G_{4}$ & $4.1 \times 10^{-3}$ & $8.9 \times 10^{-6}$ & - & 4.5 & - & 5078.98 & 4548.35 & - \\
\hline $23-32$ & $3 d^{3} G_{4}-3 d^{3} F_{4}$ & $9.8 \times 10^{-4}$ & $7.3 \times 10^{-5}$ & - & 49. & - & 2762.28 & 2790.41 & - \\
\hline $24-32$ & $3 d^{3} G_{5}-3 d^{3} F_{4}$ & $1.0 \times 10^{-3}$ & $7.8 \times 10^{-5}$ & - & 52. & - & 2941.44 & 2989.00 & - \\
\hline
\end{tabular}

Notes. The lines are grouped in three wavelength ranges, EUV, UV, and UV/visible, and are displayed in decreasing order of intensity. Columns 3, 4 show the relative line intensities (photons) Int $=N_{j} A_{j i} / N_{\mathrm{e}}$ calculated at $\log T[\mathrm{~K}]=6.11$ and electron densities of $10^{8}, 10^{12} \mathrm{~cm}^{-3}$, normalized to the intensity of the brightest line at $10^{8} \mathrm{~cm}^{-3}$. Columns 5, 6 show the $g f$ and $A$ values. Column 7 shows, for comparison, the NIST $A$ values. The last columns show the wavelengths corresponding to the experimental energies $E_{\exp }$ of Table 1, those from the scattering calculation, and those from the NIST energies.

propose the identification of the $2-51467 \AA$ line. Figure 4 shows the emissivity ratio of the $1467.06 \AA$ line from the Sandlin et al. (1977) observation $40^{\prime \prime}$ above an active region, together with those of new forbidden lines identified here, as described below.

\subsection{Transitions from the $3 s 3 p^{5}$ to the ground}

Dipole-allowed decays from $3 \mathrm{~s} 3 \mathrm{p}^{5}$ levels to the ground produce strong lines in the 300-370 ̊ range. These lines were identified for the first time using laboratory spectra by Fawcett (1971). The best spectra for the benchmark in terms of line intensities are those from the SERTS-89 and SERTS-97 rocket flights. Figure 5 shows the emissivity ratio curves, which agree to within $20 \%$ for all the lines known not to be blended. This confirms the accuracy of the scattering calculation for transitions to the $3 \mathrm{~s} 3 \mathrm{p}^{5}$ levels.

An excellent $\theta$-pinch calibrated spectrum was presented by Wang et al. (1984). Similarly, very good agreement in transitions from the $3 \mathrm{~s} 3 \mathrm{p}^{5}$ levels is found when considering their laboratory spectrum, as shown in Fig. 6. This spectrum also provides good agreement with lines from the $3 \mathrm{~s}^{2} 3 \mathrm{p}^{3} 3 \mathrm{~d}$ configuration, confirming the accuracy in the relative excitation rates between transitions from these two configurations (and the ground one).
The energy of level 6 is from the bright 1-6 observed by Be76 at $352.670 \AA$. Smitt et al. (1976) (hereafter S76) and TN94 measured $352.661 \AA 352.672 \AA$ respectively. This gives excellent agreement between predicted and observed wavelengths for the 2-6 transition observed by Be76 and TN94 at 369.161, $369.163 \AA$ A. S76 measured $369.154 \AA$, in slight disagreement. The energy of level 7 is from the strong $1-7$ transition observed at 341.112, 341.113, $341.114 \AA$ by Be76, S76, and TN94, respectively. Notice that the strong 3-7 and 2-7 transitions are predicted to be at $358.613 \AA$ and $356.519 \AA$ respectively. Also notice that S76 measured 358.621 $\AA$ and $356.519 \AA$ in laboratory spectra, and that both lines are blended in solar spectra. The energy of level 8 is from the weak 2-8 transition measured by S76 at $349.046 \AA$. This transition is severely blended with lines from Mg VI in solar spectra.

The energy of level 9 (which is highly mixed) is tentatively assigned by adopting the Be76 wavelength of $308.544 \AA$ for the 4-9 transitions, noting that this line is likely blended. Indeed TN94 in their active region spectrum measure a line with a different wavelength, $308.575 \AA$. The MR-RP energies predict $308.71 \AA$ A, while Jupén et al. (1993) identified the 4-9 with the $308.515 \AA$ line in their laboratory spectra. S76 identified 
Table 3. Summary of line identifications for Fe XI.

\begin{tabular}{|c|c|c|c|c|}
\hline$i-j$ & $\begin{array}{l}\lambda_{\exp } \\
(\AA) \\
\end{array}$ & $\begin{array}{l}\lambda_{\text {obs }} \\
(\AA) \\
\end{array}$ & ID & Diff. ID \\
\hline $6-103$ & 168.929 & ? 168.929(10) Be76 & $\mathrm{N}$ & \\
\hline $1-43$ & 178.058 & 178.056(4) Be76 & G66 & \\
\hline 4-46 & 179.758 & 179.758(10) Be76 & G66 & \\
\hline $1-42$ & 180.401 & 180.401(2) Be76 (bl) & G66 & \\
\hline $2-44$ & 180.594 & 180.595(4) Be76 & F71 & \\
\hline $3-44$ & 181.130 & 181.131(10) Be76 & G66 & \\
\hline $2-43$ & 182.167 & 182.167(2) Be76 & G66 & \\
\hline 4-45 & 184.793 & 184.793(10) Be76 (bl u) & FG66 & \\
\hline $1-38$ & 188.216 & 188.216(2) Be76 & B77 & F71 (188.299) \\
\hline $1-37$ & 188.299 & 188.299(2) Be76 & J93 & B77(189.94) \\
\hline $2-41$ & 189.123 & 189.123(4) Be76 (bl u) & B77 & J93 (192.619) \\
\hline $3-41$ & 189.711 & $189.723(5) \mathrm{N}(\mathrm{bl})$ & B77 & \\
\hline $1-36$ & 190.382 & $190.382(5) \mathrm{N}(\mathrm{bl} \mathrm{u})$ & $\mathrm{N}$ & $\operatorname{Be} 76(\mathrm{~S} \mathrm{XI})$ \\
\hline 2-39 & 192.021 & $192.021(5) \mathrm{N}(\mathrm{bl})$ & B77 & \\
\hline 3-39 & 192.627 & $192.624(5) \mathrm{N}(\mathrm{bl} \mathrm{u})$ & B77 & \\
\hline $2-38$ & 192.813 & $192.811(5) \mathrm{N}(\mathrm{bl} \mathrm{O} \mathrm{v,} \mathrm{u})$ & F71 & \\
\hline 3-37 & 193.512 & - (bl Fe XII 193.509(2)) & & \\
\hline $4-41$ & 198.538 & 198.555(10) Be76 (bl S VIII) & B77 & Be76, J93 \\
\hline $1-35$ & 201.112 & 201.112(5) N (bl Fe XIII) & $\mathrm{N}$ & \\
\hline 4-39 & 201.734 & 201.734(10) Be76 (bl Fe XII) & B77 & \\
\hline $1-34$ & 202.424 & 202.424(10) Be76 (bl u) & $\mathrm{N}$ & B77 (201.575) \\
\hline 4-38 & 202.609 & - (bl S VIII 202.608(10)) & & \\
\hline $4-37$ & 202.705 & 202.710(10) Be76 (bl) & & \\
\hline $1-30$ & 206.169 & 206.169(10) Be76 (bl u) & $\mathrm{N}$ & \\
\hline $1-29$ & 206.258 & $206.258(5) \mathrm{N}$ & $\mathrm{N}$ & \\
\hline 2-34 & 207.751 & 207.749(5) N (bl u) & $\mathrm{N}$ & \\
\hline $2-33$ & 209.771 & 209.771(5) N (bl u) & & \\
\hline $1-20$ & 234.730 & 234.73(2) D78 & $\mathrm{N}$ & D78 (Fe XV) \\
\hline $1-18$ & 236.494 & 236.494(10) Be76 & $\mathrm{N}$ & \\
\hline $1-17$ & 239.780 & ? 239.78(2) D78 & $\mathrm{N}$ & \\
\hline $1-16$ & 240.717 & 240.713(4) Be76 (bl Fe XIII) & $\mathrm{N}$ & \\
\hline $1-15$ & 242.215 & 242.215(10) (bl) Be76 & $\mathrm{N}$ & \\
\hline $4-21$ & 254.596 & $254.600(5) \mathrm{N}$ & $\mathrm{N}$ & \\
\hline $1-14$ & 256.919 & 256.925(5) N (bl Fe XII) & $\mathrm{N}$ & \\
\hline $4-20$ & 257.547 & 257.547(10) Be76 (sbl) & $\mathrm{N}$ & \\
\hline $1-13$ & 257.554 & 257.547(10) Be76 (sbl) & J93 & T98 $(257.26 \mathrm{~T})$ \\
\hline $1-12$ & 257.772 & 257.772(4) Be76 & J93 & T98 $(257.55 \mathrm{~T})$ \\
\hline $1-11$ & 257.914 & $257.914(5) \mathrm{N}$ & $\mathrm{N}$ & T98 (257.78 T) \\
\hline $4-16$ & 264.772 & bl Fe XIV 264.787 & $\mathrm{~N}$ & \\
\hline $4-15$ & 266.586 & ? 266.613(5) N (bl) & $\mathrm{N}$ & \\
\hline $21-79$ & 266.759 & 266.755(5) N (bl u) & $\mathrm{N}$ & \\
\hline $4-9$ & 308.544 & 308.544(4) (sbl) Be76 & F71 & \\
\hline $16-67$ & 308.991 & 308.991(4) В00 & $\mathrm{N}$ & \\
\hline $14-54$ & 326.323 & 326.323(4) B00 & $\mathrm{N}$ & \\
\hline $1-7$ & 341.113 & 341.112(10) Be76 & F71 & \\
\hline $2-8$ & 349.046 & 349.046(8) S76 (bl Mg VI) & F71 & \\
\hline $1-6$ & 352.670 & 352.670(10) Be76 & F71 & \\
\hline $2-7$ & 356.519 & $356.519(8) \mathrm{S} 76 \mathrm{bl}$ & F71 & \\
\hline $3-7$ & 358.613 & $358.621(8) \mathrm{S} 76 \mathrm{bl}$ & F71 & \\
\hline $2-6$ & 369.163 & 369.161(10) Be76 & F71 & \\
\hline $4-6$ & 406.822 & 406.791(4) TN94 & $\mathrm{N}$ & \\
\hline $6-21$ & 680.406 & ? bl 680.28(1) F97 & $\mathrm{N}$ & \\
\hline $6-14$ & 946.289 & 946.29(1) F97 & $\mathrm{N}$ & \\
\hline $13-32$ & 1018.90 & 1018.89(1) F97 (bl) & $\mathrm{N}$ & F97 (Ar XII) \\
\hline $14-32$ & 1028.95 & 1028.95(1) F97 (bl) & $\mathrm{N}$ & \\
\hline $16-32$ & 1408.71 & 1408.70(1) F97 & $\mathrm{N}$ & \\
\hline $13-25$ & 1409.44 & $1409.45(1)$ S77 & $\mathrm{N}$ & \\
\hline $14-25$ & 1428.76 & 1428.75(1) S77 & $\mathrm{N}$ & \\
\hline $2-5$ & 1467.07 & 1467.06(2) S77 & J71 & \\
\hline
\end{tabular}

instead the 5-9 transition with a laboratory line observed at $355.837 \AA$. An energy of 361859 would follow, and a wavelength of $308.532 \AA$ for the 4-9 transition is predicted, not too far from the Be76 wavelength.
Table 3. continued.

\begin{tabular}{cllll}
\hline \hline$i-j$ & $\begin{array}{l}\lambda_{\exp } \\
(\AA)\end{array}$ & $\begin{array}{l}\lambda_{\text {obs }} \\
(\AA)\end{array}$ & ID & Diff. ID \\
\hline $14-24$ & 1582.55 & $1582.56(2)$ S77 & $\mathrm{N}$ & FD77,S77 \\
$14-23$ & 1639.77 & $1639.78(3)$ S77 (bl O VII) & $\mathrm{N}$ & S77 \\
$1-4$ & 2649.50 & $2648.71(2)$ S77 (air) & S77 & \\
$2-4$ & 3988.00 & $3986.8(5)$ Je71 (air) & & \\
$21-24$ & 4567.46 & $4566.2(5)$ Je71 (air) & MN77 & \\
$1-2$ & 7894.03 & $7891.8(1)$ Je71 (air) & & \\
\hline
\end{tabular}

Notes. The columns indicate: 1) the indices corresponding to Table 1; 2 ) the wavelengths calculated from the experimental energies $E_{\exp }$; 3 ) the observed wavelengths $\lambda_{\text {obs }}$. The original source is indicated. $\mathrm{N}$ refers to a new measurement presented here. For lines above $2000 \AA$, air wavelengths are indicated. bl, sbl indicate that a line is blended or is a self-blend. $\mathrm{u}$ indicates blending with an unidentified line. Columns 4) and 5) indicate some of the previous identifications consistent or not with the present ones (with observed wavelengths in $\AA$ ). $\mathrm{N}$ in Col. 4 indicates a new identification.

References. G66 = Gabriel et al. (1966); F67 = Fawcett et al. (1967); F70 = Fawcett (1970); F71 = Fawcett (1971); Je71 = Jefferies et al. (1971); J71 = Jordan (1971); MN77 = Mason \& Nussbaumer (1977); S76 = Smitt et al. (1976); Be76 = Behring et al. (1976); SBT77 = Sandlin et al. (1977); FD77 = Feldman \& Doschek (1977); B77 = Bromage et al. (1977); D78 = Dere (1978); J93 = Jupén et al. (1993); TN94 = Thomas \& Neupert (1994); F97 = Feldman et al. (1997); T98 = Träbert (1998).

\subsection{Transitions from the $3 s^{2} 3 p^{3} 3 d$ configuration to the ground}

Dipole-allowed decays from $3 s^{2} 3 p^{3} 3 d$ levels to the ground produce the brightest Fe XI lines, visible in the EUV in the 160-260 A range (see Table 2). Several lines were first identified by Gabriel et al. (1966). More identifications were provided by Bromage et al. (1977); however, many subsequent authors have provided differing identifications (e.g. Jupén et al. 1993; Träbert 1998).

\subsection{1. $3 s^{2} 3 p^{3}\left({ }^{4} S\right) 3 d^{5} D_{j}^{\circ}$ (levels $\left.10-14\right)$}

These levels are relatively pure. As shown by Träbert (1998, and references therein), the transition array $3 \mathrm{~s}^{2} 3 \mathrm{p}^{4}{ }^{3} \mathrm{P}_{j}^{\mathrm{e}}-3 \mathrm{~s}^{2} 3 \mathrm{p}^{3} 3 \mathrm{~d}^{5} \mathrm{D}_{j}^{\circ}$ produces two groups of lines that are strong in the delayed beam-foil spectra. The first group consists of transitions to the $J=2$ ground state and is observed around $257 \AA$. The second group consists of decays to the $J=0,1$ levels, and is observed around $266 \AA$. In astrophysical conditions, our calculations predict that the strongest decays from the $3 \mathrm{~s}^{2} 3 \mathrm{p}^{3} 3 \mathrm{~d}^{5} \mathrm{D}_{j}^{\circ}$ levels are the $1-14,1-13,1-12$, and $1-11$, in order of decreasing intensity. The beam-foil spectra are very important because they provide a tight constraint on the possible energies of the $3 \mathrm{~s}^{2} 3 \mathrm{p}^{3} 3 \mathrm{~d}^{5} \mathrm{D}_{j}^{\circ}$ levels, although the spectral resolution is such that wavelengths are only known with an accuracy of $1-2 \AA$.

In the first group of transitions, Träbert (1998) tentatively identified the $1-13,1-12$, and 1-11 transitions with the lines observed at 257.26, 257.55, $257.78 \AA$, respectively. Different identifications are provided here, mainly based on the Hinode/EIS observations as described below. Regarding the second group of transitions, Träbert (1998) tentatively identified the $2-12,2-11$, and $2-10$ with the lines observed at 266.23, 

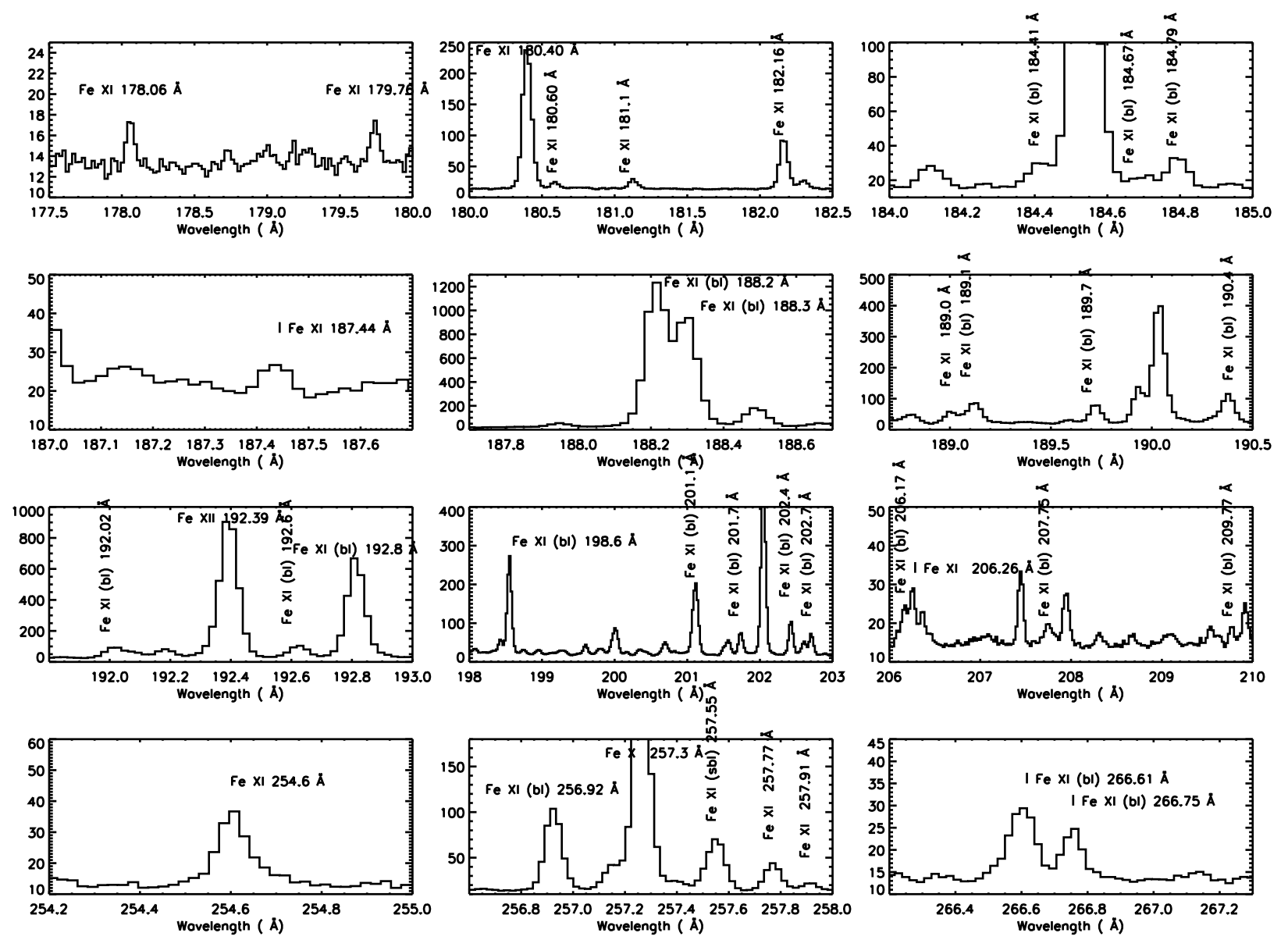

Fig. 3. Sections of the Hinode EIS off-limb spectrum used to benchmark Fe XI (units are averaged counts per pixel).

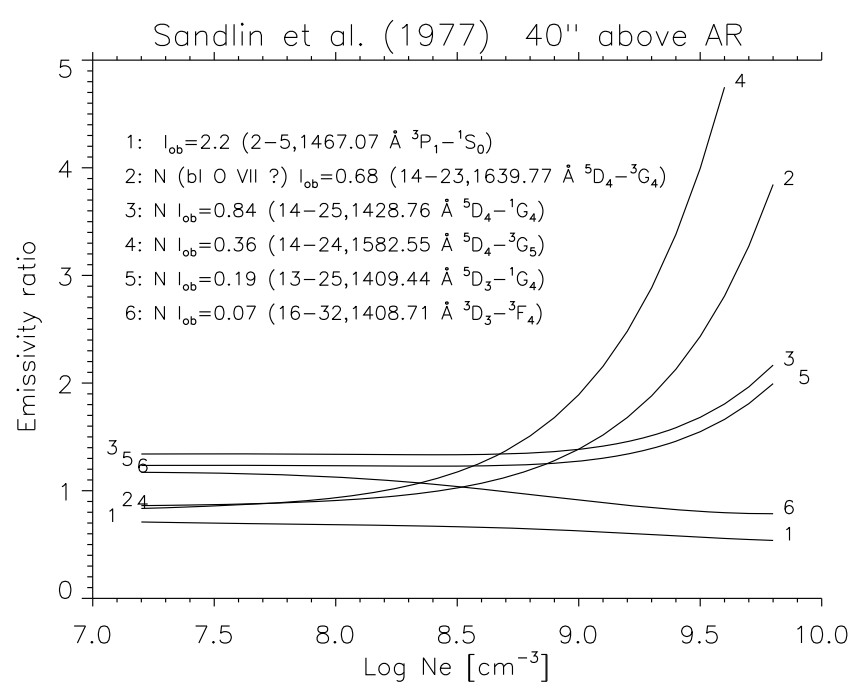

Fig. 4. The emissivity ratio curves from the Skylab ATM observation 40" above an active region reported by Sandlin et al. (1977). $I_{\mathrm{ob}}$ indicates the measured line intensity. Lines newly identified are indicated by $\mathrm{N}$.

266.42, and $266.60 \AA$. These lines are predicted to be extremely weak in solar spectra and might actually not be observable. The energy of level 10 is therefore very difficult to assign accurately. However, using the the firm identifications of the other ${ }^{5} \mathrm{D}_{j}^{\circ}$ levels and their theoretical splittings (see below), a wavelength of $266.74 \AA$ for the $2-10$ transition is predicted. This transition is tentatively assigned to a weak line observed with Hinode/EIS at $266.755 \AA$, with a wavelength still consistent with the beamfoil measurements. The tentative identification by Träbert (1998) is therefore ruled out.

The energy of level 11 is assigned from the weak 1-11 transition, which is identified here with the Hinode/EIS line at $257.914 \AA$. This new energy predicts a wavelength of $266.63 \AA$ for the 2-11 transition, which is different from (though very close to) the suggested value of $266.42 \AA$ given by Träbert (1998). The energy of level 12 is obtained by assigning the observed wavelength of $257.772 \AA$ to the $1-12$ transition. This agrees with the identification given by Jupén et al. (1993). We find excellent agreement between the Be76 and Hinode/EIS wavelength. The new energy predicts a wavelength of $266.47 \AA$ for the $2-12$ transition, which is different from (though very close to) the suggested value of $266.23 \AA$ given by Träbert (1998). Similarly, the energy of level 13 is obtained by assigning the observed wavelength of $257.55 \AA$ to the $1-13$ transition, in agreement with the identification given by Jupén et al. (1993). However, this line is actually a close self-blend with the 4-20 transition (see below). The wavelength given by Be76 is $257.547 \AA$, which provides an energy of 388279 . The actual energy chosen here is 388268 , based on 4 new identifications of strong UV lines that form two branching ratios, the $14-25$ vs. 13-25 and the 14-32 vs. the 13-32 line (see below).

The model ion predicts a strong decay to the ground state from level 14, observable by Hinode/EIS. It is identified here with the strong line observed by EIS at $256.925 \AA$. However, the energy of this level is obtained from the new identification of 

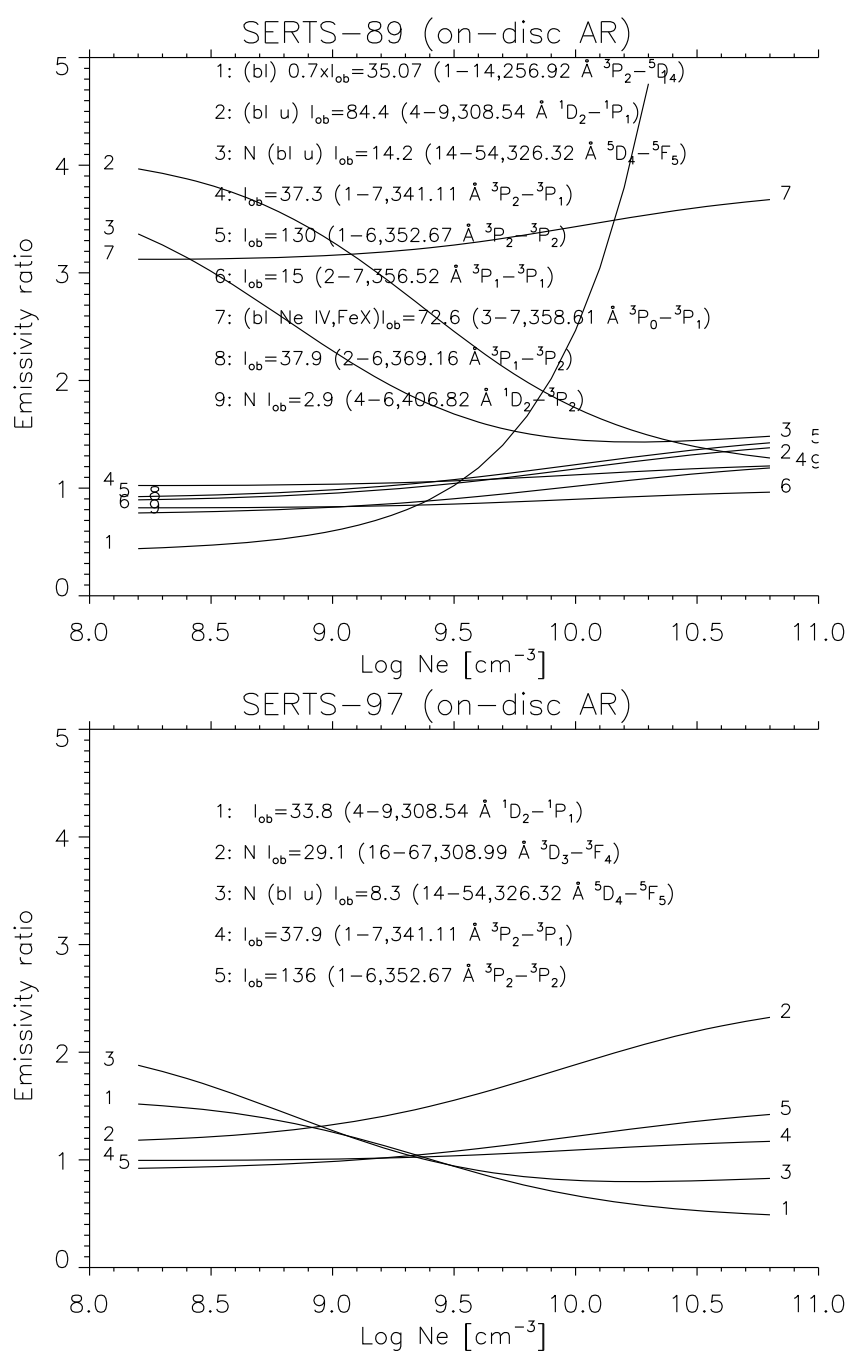

Fig. 5. Emissivity ratio curves relative to the SERTS-89 and SERTS-97 calibrated data.

the 6-14 transition, observed by SOHO/SUMER at 946.29. The energy of level 14 provides a wavelength for the decay to the ground of $256.920 \AA$, close to the EIS value. The energy agrees with the observed wavelength of the 14-32 UV transition, as described below. Be76 also reports $256.925 \AA$ A, but gives a tentative identification as an unspecified $\mathrm{Fe} X \mathrm{X}$ transition. In reality, current atomic models for Fe XV do not predict any strong line due to this ion around these wavelengths. Also, in the EIS offlimb quiet-Sun spectra there is almost no Fe XV emission, so any blending with this ion can be ruled out. Del Zanna \& Mason (2005) tentatively identified a level in Fe XII that would provide a weak transition around $256.93 \AA$. However, the Fe XII atomic data predict that this line would only be $2-3 \%$ the intensity of this strong transition for the Hinode/EIS spectrum considered here. Indeed, the predicted intensity is within the observed one, as Fig. 7 (top right) shows.

The decays from the $3 \mathrm{~s}^{2} 3 \mathrm{p}^{3} 3 \mathrm{~d}^{5} \mathrm{D}_{j}^{\circ}$ levels are particularly important because they provide a direct temperature diagnostic, when observed in combination with any of the other lines from higher levels, as shown in Fig. 7 (top left). To within 30\%, the observed lines indicate an isothermal electron temperature of $\log T[\mathrm{~K}]=5.8$, perhaps lower than expected. The absolute value depends on the relative calibration between the two EIS channels which is not well established. The lines presented in Fig. 7

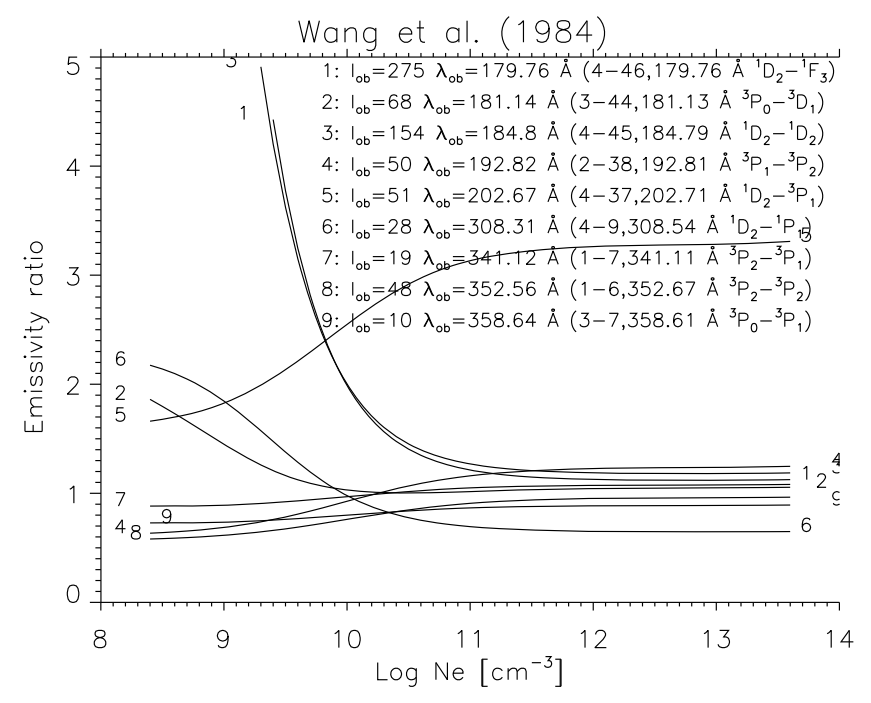

Fig. 6. Emissivity ratio curves relative to the laboratory spectrum of Wang et al. (1984). $\lambda_{\mathrm{ob}}$ indicates the observed wavelength.

(top left) depend very little from the electron density, so the resulting temperature does not depend on the density. On the other hand, many of the lines discussed in this section have some temperature dependence. The value of $\log T[\mathrm{~K}]=5.8$ was used for the emissivity ratio curves (as a function of density) for the other lines shown in Fig. 7. Larger discrepancies are found in general if a temperature close to $\log T[\mathrm{~K}]=6.11$ is used.

\subsection{2. $3 s^{2} 3 p^{3}\left({ }^{2} D\right) 3 d^{3} D_{j}^{\circ}$ (levels $15-17$ )}

The 15-17 are highly mixed levels. The model ion predicts that two decays from level 16 should be observable. That one to the ground state is not visible by Hinode/EIS, but the 4-16 should be an observable line. A search through possible wavelength coincidences produces only one result: the 1-16 transition should be blended with Fe XIII in the line observed at $240.713 \AA$ by Hinode/EIS, while the 4-16 line would be blended with a strong Fe XIV line observed at $264.787 \AA$. That the $240.713 \AA$ line is blended is confirmed by two facts. First, Be76 indicate that the line is a blend, and TN94 report a very broad line, with $0.102 \AA F W H M$. Second, the Fe XIII benchmark (work in progress) indicates that about half of the intensity of this line does not come from Fe XIII. The new identification (see below) of the $1408.70 \AA$ line with the 16-32 transition provides an accurate energy for level 16 of 415426 , which results in a wavelength of $240.717 \AA$, which is only $4 \mathrm{~m} \AA$ from the observed blend.

Levels 15 and 17 are more difficult to establish. The 1-15 transition should be well observable. Based on the differences between theoretical and observed energies of the other levels, this transition is predicted to be at $242.2 \AA$. Be76 measured an unidentified line at $242.215 \AA$, so we identify it with the 1-15 transition, although its observed intensity is about twice the predicted one. This predicts that a weak but observable 4-15 line by EIS should fall at $266.586 \AA$. There is an unidentified line, with about the right intensity, but at $266.613 \AA$. The same arguments apply to level 17 , with a decay to the ground state predicted to have a wavelength of $239.75 \AA$. This line should be much weaker than the $1-15$, so it is likely to be either the $239.52 \AA$ observed by Behring et al. (1976) or the $239.78 \AA$ observed by Dere (1978). We tentatively assign it to the latter. 

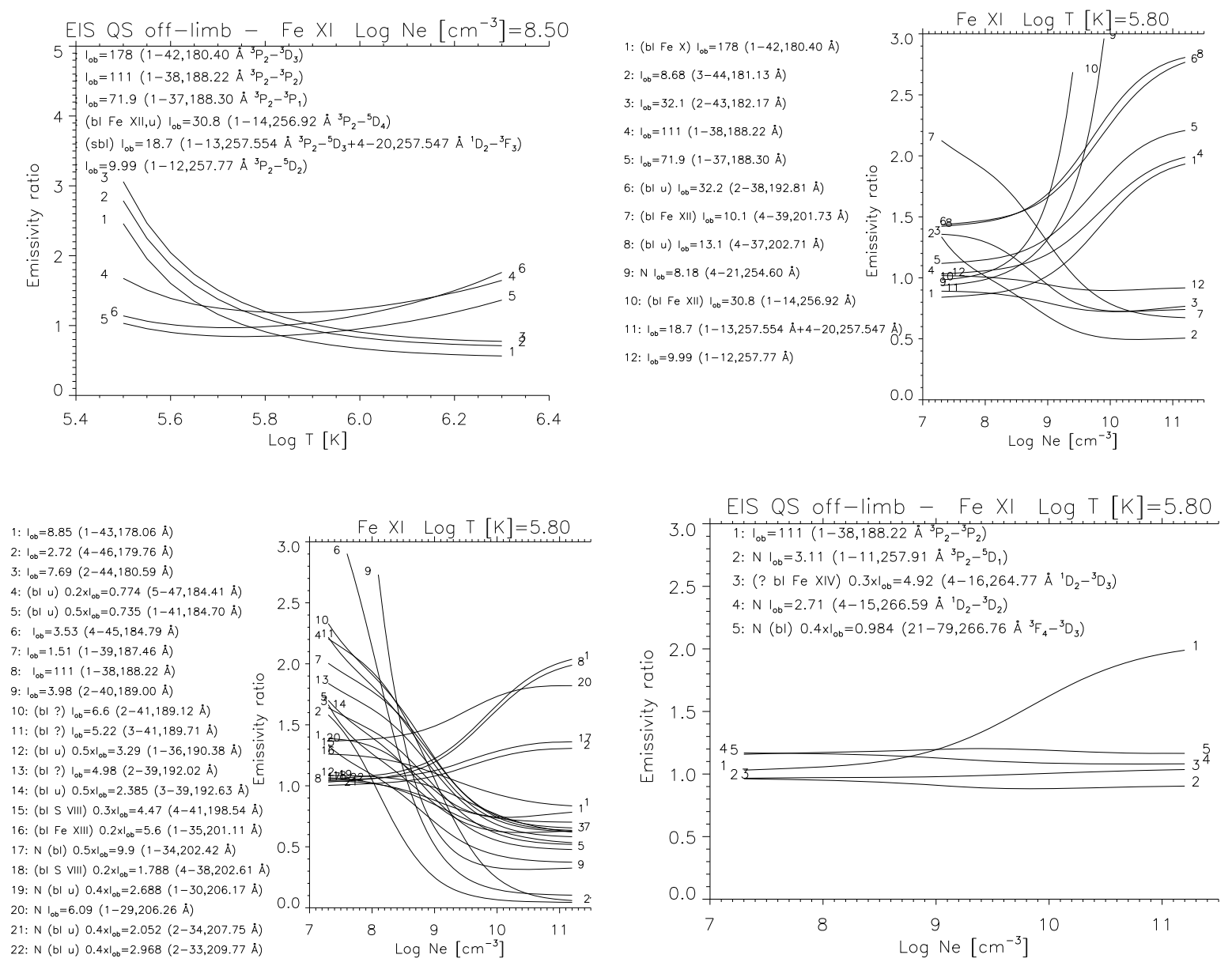

Fig. 7. Emissivity ratio curves for a selection of lines from the Hinode EIS off-limb spectrum. In the top left, the curves are shown as a function of the electron temperature, the others as a function of density. The top right plot shows the curves for the brightest lines, while the others include the weaker ones, in the SW and LW channels.

\subsection{3. $3 s^{2} 3 p^{3}\left({ }^{2} D\right) 3 d^{3} F_{j}^{\circ}$ (levels $\left.18,20,21\right)$}

Levels 18, 20, 21 are relatively pure. The energy of the important metastable level 21 is obtained here from the Be76 measurement of the previously-unidentified line at $254.596 \AA$ with the strong 4-21 transition. The Hinode/EIS wavelength is $254.600 \AA$. This is a new identification. The new energy for this level predicts that the strong 6-21 transition would be at $680.40 \AA$. There is indeed a strong, previously unidentified, coronal line blending a line observed by SUMER at $680.28 \AA$. The forbidden 14-21 transition is predicted to have a wavelength of $2423 \AA$ (vacuum), and it was too weak to be observed by Sandlin et al. (1977).

The energy of level 20 is obtained from the following considerations. The theoretical splitting with level 21 predicts that a weak but observable line should be present around $257.51 \AA$. The transitions 4-20 and 1-20 are predicted to be weak, however the 4-20 in particular should be observable with EIS. If we identify it as blending (with the 1-13) the line observed at $257.547 \AA$, we obtain a wavelength for the decay to the ground state of $234.73 \AA$, which coincides with a line reported as Fe XV by Dere (1978). To support this, it appears unlikely that the $234.73 \AA$ line comes from Fe XV. The Fe XV $3 s 3 p^{3} P_{2}-3 s 3 d^{3} D_{2}$ line is in fact predicted to be more than ten times weaker than the $3 \mathrm{~s} 3 \mathrm{p}^{1} \mathrm{P}_{1}-3 \mathrm{~s} 3 \mathrm{~d}^{1} \mathrm{D}_{2}$ transition observed at $243.78 \AA$, while the observed intensity is approximately half or more (if blending with Ar XV in the $243.78 \AA$ line is considered).
Having established the energies of the other levels, it becomes easier to find the energy of level 18 . Theoretical splittings predict that an observable line should fall around $236.5 \AA$. Indeed there is an unidentified line in the Be76 spectrum at $236.494 \AA$.

\subsection{4. $3 s^{2} 3 p^{3}\left({ }^{2} D\right) 3 d^{3} G_{i}^{\circ}$ (levels 22-24)}

Levels 22-24 are relatively pure. The energy of the important metastable level 24 is obtained from the 14-24 transition, which is identified here as the coronal line observed by Sandlin et al. (1977) at $1582.56 \AA$ and from the new energy of the level 14. Excellent agreement in terms of line intensity is found, as shown in Fig. 4. Sandlin et al. (1977) gave a tentative identification as Ar XIII for this line. The new energy predicts an air wavelength of $4566.2 \AA$ for the strong 21-24 transition, exactly the same value measured by Jefferies et al. (1971). Mason \& Nussbaumer (1977) originally suggested this identification. Feldman \& Doschek (1977) incorrectly identified the coronal line at $1582.6 \AA$ with an Fe X transition. As shown by Del Zanna et al. (2004), the Fe X transition was predicted to be more than two orders of magnitude weaker than the observed line.

The energy of the important metastable level 23 is obtained as follows. Having established the energy of level 24, the theoretical splitting between levels 23 and 24 predicts that a relatively strong 14-23 transition should be observed around 
1: (dbl) $\mathrm{I}_{\mathrm{OD}}=2 \lambda_{\mathrm{ob}}=178 \AA(1-43,178.06 \dot{A})$

2: $\mathrm{I}_{\mathrm{ob}}=59 \lambda_{\mathrm{ob}}=180.4 \dot{A}(1-42,180.40 \dot{A})$

3: $(\mathrm{dbl}) \mathrm{I}_{\mathrm{ob}}=2 \lambda_{\mathrm{ob}}=180.7 \dot{A}(2-44,180.59 \dot{A})$

4: $\mathrm{I}_{\mathrm{ob}}=2.7 \lambda_{\mathrm{ob}}=181.1 \dot{A}(3-44,181.13 \dot{A})$

5: $\mathrm{I}_{\mathrm{ob}}=10.3 \lambda_{\mathrm{ob}}=182.2 \AA(2-43,182.17 \dot{A})$

6: (sbl) $\mathrm{I}_{\mathrm{ob}}=45.6 \lambda_{\mathrm{ob}}=188.2 \dot{A}\left(1-38,188.216 \dot{A}+1-37,188.2{ }_{9} Q_{A}\right)$

7: (bl OV) $\left.\right|_{o b}=7.8 \lambda_{o b}=192.8 \AA(2-38,192.81 \AA)$

8: $* \mathrm{~N}_{\mathrm{ob}}=1.6 \lambda_{\mathrm{ob}}=234.7 \AA(1-20,234.73 \AA)$

9: $* \mathrm{~N} \mathrm{I}_{\mathrm{ob}}=1.8 \lambda_{\mathrm{ob}}=236.5 \dot{A}(1-18,236.49 \dot{A})$

10: $* \mathrm{~N}\left(\mathrm{bl} \mathrm{Fe} \mathrm{XIII)} \mathrm{I}_{\mathrm{ob}}=4.6 \lambda_{\mathrm{ob}}=240.8 \dot{A}(1-16,240.72 \dot{A}) \cdot \frac{0}{\varepsilon}\right.$

11: *N Iob $=1.5 \lambda_{\mathrm{ob}}=242.2 \AA(1-15,242.21 \dot{A})$

12: $* N \mathrm{I}_{\mathrm{ob}}=2 \quad \lambda_{\mathrm{ob}}=254.7 \dot{A}(4-21,254.60 \dot{A})$

13: $* N \mathrm{I}_{\mathrm{ob}}=4.6 \lambda_{\mathrm{ob}}=256.9 \dot{A}(1-14,256.92 \dot{A})$

14: $\left.*\right|_{o b}=3.4 \lambda_{o b}=257.6 \AA(1-13,257.55 \AA)$

15: * $\left.\right|_{\mathrm{ob}}=1.2 \quad \lambda_{\mathrm{ob}}=257.8 \AA(1-12,257.77 \AA)$

16: *N Iob $=0.7 \lambda_{\mathrm{ob}}=266.8 \dot{A}(4-15,266.586 \dot{A}+21-79,266.759 \AA)$

0.0

7
From Malinovsky \& Heroux (1973)

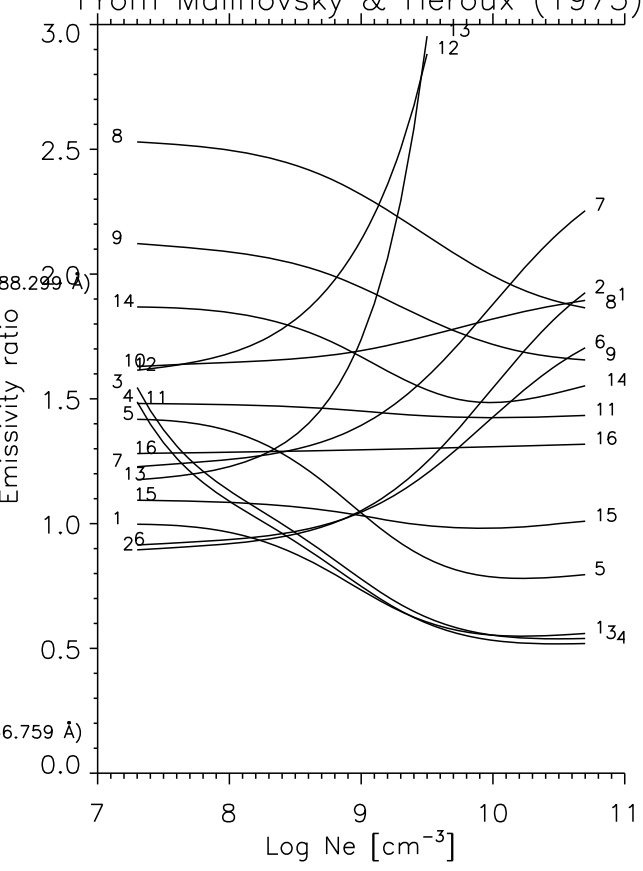

Fig. 8. The emissivity ratio curves relative to the full-Sun spectrum of Malinovsky \& Heroux (1973). Lines deblended (dbl) and blended (bl, sbl) are indicated. Those with a * were not published and have a large (30-50\%) uncertainty. Lines newly identified are indicated by $\mathrm{N}$.
1639 Å. Indeed, Sandlin et al. (1977) reported a line observed at $1639.78 \pm 0.03 \AA$, identified by them as the O VII 1s $2 \mathrm{~s}{ }^{3} \mathrm{~S}_{1}-1 \mathrm{~s} 2 \mathrm{p}{ }^{3} \mathrm{P}_{0}$ transition. This transition, however, is predicted to be 5 times weaker than the $1 \mathrm{~s} 2 \mathrm{~s}{ }^{3} \mathrm{~S}_{1}-1 \mathrm{~s} 2 \mathrm{p}{ }^{3} \mathrm{P}_{2}$ transition observed by Sandlin et al. (1977) at $1623.54 \AA$. In their actual off-limb spectra, the $1639.78 \AA$ line was instead more than twice as strong as the $1623.54 \AA$ line. The 14-23 transition is therefore identified as blending the O VII 1s $2 \mathrm{~s}{ }^{3} \mathrm{~S}_{1}-1 \mathrm{~s} 2 \mathrm{p}{ }^{3} \mathrm{P}_{0}$ line. Actually, in the off-limb spectrum, it appears that the observed line is entirely due to Fe XI, as shown in Fig. 4. The decay 13-23 is predicted to be 10 times weaker, at $1614.39 \AA$, possibly blending the S XI measured at $1614.51 \AA$ by Sandlin et al. (1977).

\subsubsection{Level 25}

The energy of the important metastable level 25 is obtained from the 14-25 transition, which is identified here for the first time as the strong coronal line, observed by Sandlin et al. (1977) at $1428.75 \pm 0.01 \AA$ A. Excellent agreement between predicted and observed intensity is found, as shown in Fig. 4. The same line is prominent in SOHO/SUMER spectra at the same wavelength (Feldman et al. 1997). This allows another new identification: the strong 13-25 transition with the line observed by Sandlin et al. (1977) (and also by Feldman et al. 1997) at $1409.45 \pm$ $0.01 \AA$. This wavelength is used to obtain the energy of level 13 , 388268 . The observed intensity is in good agreement with the predicted one also in this case, as Fig. 4 shows.

\subsection{6. $3 s^{2} 3 p^{3}\left({ }^{2} P\right) 3 d^{3} P_{j}^{\circ}$ (levels $\left.28,29,34\right)$}

Levels 28, 29, and 34 are relatively pure. The strongest decay is the 1-34 transition. We identify it as the (previously unknown) $202.424 \AA$ line in the Behring et al. (1976) spectrum. The EIS wavelength is very close, $202.426 \AA$. The weaker
2-34 transition is therefore predicted to be at $207.751 \AA$. Indeed a weak line in the Hinode EIS spectra is observed at $207.749 \AA$.

The $3 \mathrm{~s}^{2} 3 \mathrm{p}^{3}\left({ }^{2} \mathrm{P}\right) 3 \mathrm{~d}^{3} \mathrm{P}_{1}^{\circ}(29)$ is predicted to produce a decay to the ground state also observable by Hinode/EIS. An inspection of the EIS spectra shows that this transition can only be one of the lines observed at 206.166, 206.258, 206.365 $\AA$ with EIS. The Be76 measurements are consistent $(206.169,206.253$, $206.369 \AA$ ). The latter is a weak Fe XII transition according to the Del Zanna \& Mason (2005) model ion. Various authors (e.g. Brown et al. 2008) identify the $206.258 \AA$ line with the (strong in relative terms) $2 \mathrm{~s}^{2}{ }^{1} \mathrm{~S}_{0}-2 \mathrm{~s} 2 \mathrm{p}^{1} \mathrm{P}_{1}$ resonance line from K XVI at $206.253 \AA$; however, this cannot be, given that the peak abundance in ionization equilibrium for this ion is $5 \mathrm{MK}$, far too high for any quiet Sun plasma. The 1-29 transition is identified here with the 206.258 $\AA$ line, considering that the 206.166 $\AA$ line is assigned to the 1-30 (see below).

\subsection{7. $3 s^{2} 3 p^{3}\left({ }^{2} P\right) 3 d^{3} F_{j}^{\circ}($ levels $30-32)$}

Among the levels 30-32, the middle one is significantly mixed and does not produce any strong transitions, so that it remains unidentified. Level 32 is a metastable level, so is important because it produces strong forbidden lines in the UV. The calculations show that a strong 14-32 line should be present in the SUMER spectra. We identify it with the (previously unidentified) coronal line observed by Feldman et al. (1997) at $1028.95 \AA$ (with a possible additional blend). This provides an energy for level 32 of 486413 (using the new energy for level 14 of 389227 ).

The line observed by SOHO/SUMER at $1018.89 \AA$ cannot be the ArXII $2 \mathrm{~s}^{2} 2 \mathrm{p}^{3}{ }^{4} \mathrm{~S}_{3 / 2}-2 \mathrm{~s}^{2} 2 \mathrm{p}^{3}{ }^{2} \mathrm{D}_{5 / 2}$ transition as identified by Feldman et al. (1997), considering that it is much brighter than the $2 \mathrm{~s}^{2} 2 \mathrm{p}^{3}{ }^{4} \mathrm{~S}_{3 / 2}-2 \mathrm{~s}^{2} 2 \mathrm{p}^{3}{ }^{2} \mathrm{D}_{3 / 2}$ (observed at $1054.57 \AA$ ), the opposite of what it should be. Its morphology suggests that it is a strong coronal line. It is identified here as the 
Fe XI 13-32 transition (possibly slightly blended with Ar XII). This provides the energy of level 13.

The energy of level 32 also provides an accurate energy for level 16. The 16-32 line is predicted to be about $1 / 2$ of the intensity of the 13-25 transition. The 16-32 line is identified here with the line observed by SOHO/SUMER at $1408.70 \AA$ (Feldman et al. 1997), based on wavelength and intensity arguments. Excellent agreement between predicted and observed intensity is found, as shown in Fig. 4.

Having established the energy of level 32, it becomes easier to find the energy of level 30, considering the theoretical splitting between the two levels. A weak 1-30 transition should be observable by Hinode/EIS, and indeed a line with the right wavelength and intensity is found at $206.166 \AA$. The Be76 measurement of $206.169 \AA$ is chosen to establish the energy of level 30 .

\subsection{8. $3 s^{2} 3 p^{3}\left({ }^{2} P\right) 3 d^{3} D_{j}^{\circ}$ (levels $\left.27,33,35\right)$}

Levels 27, 33, and 35 are mixed, in particular the middle one. The strongest transition is the $1-35$, predicted to be around $201 \AA$. When considering its intensity, the only possibility is that this transition is blending a much stronger Fe XIII line. The blend is at $201.112 \AA$ as measured by Hinode/EIS. The line is slightly broader than the others, which also confirms blending. Be76 measured 201.121 A but indicated a blend with a secondorder line. Having established level 35, it follows that level 33 should produce a transition (2-33) observable by Hinode/EIS around $209.8 \AA$. Indeed an unidentified line at $209.771 \AA$ is observed, and this wavelength is used to establish the energy of level 33. Level 27 remains unknown, given that it produces a weak $3-27$ transition around $214 \AA$, not observable by Hinode/EIS.

\subsubsection{Level 36}

The weak 1-36 transition is identified here with a blend observed by Hinode/EIS at $190.382 \AA$. Various authors (e.g. Be76, Brown et al. 2008) have previously identified a line observed around $190.4 \AA$ with the S XI $2 \mathrm{~s}^{2} 2 \mathrm{p}^{2}{ }^{1} \mathrm{D}_{2}-2 \mathrm{~s} 2 \mathrm{p}^{3}{ }^{1} \mathrm{P}_{1}$, which has a different wavelength of $190.355 \AA$, according to NIST. Be76 measured it at $190.372 \AA$. This transition cannot be clearly due to $\mathrm{S}$ XI for three reasons. First, this ion emits around $2 \mathrm{MK}$, too high a temperature for the quiet Sun. Second, the intensity of this transition is predicted to be only about $3 \%$ of the intensity of the S XI $2 \mathrm{~s}^{2} 2 \mathrm{p}^{2}{ }^{3} \mathrm{P}_{1}-2 \mathrm{~s} 2 \mathrm{p}^{3}{ }^{3} \mathrm{~S}_{1} 188.675 \AA$, which is observed in the off-limb EIS spectrum at $188.673 \AA$ to be very weak. Third, its wavelength does not coincide with the NIST wavelength for S XI.

\subsubsection{Levels $37-41$}

A detailed account of the complexities in establishing the identifications of these levels is provided in Del Zanna et al. (2010). The energy of level 38 is obtained from the strong 1-38 transition, observed by Be76 at $188.216 \AA$. The energy of level 37 is obtained from the strong 1-37 transition, observed by Be76 at $188.299 \AA$. The level has another strong decay (4-37) with a predicted wavelength of $202.706 \AA$, in good agreement with the observed one by Behring et al. (1976) and Hinode/EIS of $202.710 \AA$. The 2-37 transition is particularly important because it blends the resonance line of Ca XVII, together with other transitions, mostly from $\mathrm{O} v$. The off-limb Hinode/EIS spectrum is particularly good because all cooler emission is absent, so this line should mainly come from Fe XI. In reality, as Fig. 7 shows, this line still appears to be blended. The line is predicted at $192.813 \AA$ and observed at $192.811 \AA$, in excellent agreement.

The 1-37 line has been the subject of much confusion and controversy, as reviewed in Del Zanna et al. (2010), with conflicting identifications and very different $g f$ values. It is therefore important to assess whether the new scattering calculations provide good agreement with observations. The best observation is by Malinovsky \& Heroux (1973), although the 1-37 was blended with the 1-38 transition in that spectrum. The agreement between the self-blend 1-38+1-37 and the other strong lines $(1-43,1-42,2-44,3-44)$ in the Malinovsky \& Heroux (1973) observation is excellent (to within 10\%). This result shows that the new scattering calculation is very accurate, because it predicts a correct intensity (to within 10\%) for the self-blend 1-38+1-37. Large discrepancies are present if any other scattering calculation is adopted. Hinode/EIS does resolve the 1-38 and 1-37 lines, and good agreement (to within 10\%) is found in the relative intensities of these two lines, as shown in Fig. 7. These lines are the strongest ones after those of Fe XII in EIS spectra (cf. Fig. 3).

The energy of level 39 is difficult to establish accurately, given that all the decays are weak and blended to some degree. The strongest decay is the 4-39 transition, blended (by about 10\%) with an Fe XII line (Del Zanna \& Mason 2005), and observed by Be76 at $201.734 \AA$, in agreement with the Hinode/EIS wavelength of $201.737 \AA$. We used the $201.734 \AA$ value to obtain the energy. This predicts that the decay to the ground state should be observed at 187.461 $\AA$. In the Hinode/EIS observation, a weak line is observed at $187.437 \AA$, which is presumably the 1-39 (blended). The 2-39 transition is predicted at $192.021 \AA$, and indeed Hinode/EIS observes a weak line exactly at $192.021 \AA$ with about the expected intensity. This line in on-disk observations is strongly blended with Fe VIII and other unidentified cool emission (Del Zanna 2009a,b), and also in flaring conditions with the strong Fe XXIV 192.028 $\AA$. The 3-39 transition is predicted at $192.627 \AA$. Hinode/EIS observes a line at the right wavelength, $192.624 \AA$, but the model predicts that only about $1 / 2$ of the intensity comes from Fe XI.

Level 40 only has one one dipole-allowed transition to the ground configuration, the 2-40 transition, identified with the line observed at $188.997 \AA$ by Behring et al. (1976) (and at $189.005 \AA$ with Hinode/EIS). Level 41 is difficult to establish because it produces various weak decays, all of which appear to be blended to some degree (see Fig. 7). The strongest one is the 4-41 line, which is blended with a strong S VIII transition observed at $198.552 \AA$ by Hinode/EIS. Be76 incorrectly identifies the line as blended with Fe XII. The second strongest line is the $2-41$, observed by Hinode/EIS at $189.121 \AA$. This line appears to be slightly blended. The Be76 wavelength of $189.123 \AA$ is chosen to establish the energy of this level. This predicts the $4-41$ to be at $198.539 \AA$, and the weaker $3-41$ transition at $189.712 \AA$. Hinode/EIS observes a (broad) line at a close wavelength, $189.723 \AA$, but slightly brighter than expected, an indication of a blend here. Be76 gives a slightly longer wavelength, $189.733 \AA$. The very weak $1-41$ is then predicted at $184.698 \AA$, a wavelength too far from the line observed by Hinode/EIS at $184.796 \AA$ (Be76 gives $184.793 \AA$ ) and identified as the 4-45 (see below). Hinode/EIS does observe some 
emission at $184.70 \AA$ with the correct intensity, although it is too weak to produce a clean line profile.

\subsubsection{Levels $42-48$}

The energy of level 42 is from the strong 1-42 line, observed at $180.401 \AA$ by Be76. This line is slightly blended with Fe X (Paper I) and Fe XII. The energy of level 43 is from the strong 2-43 transition observed at $182.167 \AA$ by Be76. This predicts the 1-43 line at $178.058 \AA$, in excellent agreement with the observed value (178.056 ̊). Hinode/EIS wavelengths are within a few $\mathrm{m} \AA$.

The energy of level 44 is provided from the wavelengths of the 3-44 and 2-44 lines, $181.131 \AA$ and $180.595 \AA$. The energy of level 45 is obtained from the weak 4-45 line, observed at $184.793 \AA$ by Be76 (184.796 $\AA$ by Hinode/EIS). The energy of level 46 is obtained from the weak 4-46 line, observed at $179.758 \AA$ by Be76. The Hinode/EIS wavelength is slightly lower $(179.742 \AA)$. The 4-45 and 4-46 lines are very strong in laboratory spectra, with wavelengths that confirm those of Be76 (B.C. Fawcett, plate C50, priv. comm.).

The energy of level 47 is provided from the wavelength of the 5-47 line, strong only in laboratory spectra. This line is very close to the strong Fe X (Paper I) line, measured by Be76 at $184.536 \AA \pm 0.002 \AA$. The wavelength measurements in second order of plate C50 (B.C. Fawcett, priv. comm.) of these two close lines provide a wavelength of $184.410 \AA \pm 0.002 \AA$, which is adopted here. Hinode/EIS observes a weak line at $184.413 \AA$, but its intensity is higher than predicted.

Finally, the ratios of the strong lines 2-43,3-44 with e.g. the $1-42$ is a well-known and very good electron density diagnostic. The curves form the Malinovsky \& Heroux (1973) observation indicate an electron density $\log N_{\mathrm{e}}=8.5 \mathrm{~cm}^{-3}$, in excellent agreement with the values derived from other ions such as Fe XII (Storey et al. 2005; Del Zanna \& Mason 2005). Good agreement is also found in Hinode/EIS spectra, although the strongest line, the 1-42 $180.40 \AA$, consistently has an observed intensity that is too low by a $20 \%$ or so (cf. Fig. 7 ). This can only be due to an incorrect radiometric calibration around $180 \AA$.

\subsection{Transitions from $3 s 3 p^{4} 3 d$}

The decays from the $3 \mathrm{~s} 3 \mathrm{p}^{4} 3 \mathrm{~d}$ configuration are predicted to be weak. The strongest transitions are the 16-67, 21-79, 6-103, and 14-54. The strongest is the 16-67, which should be observable and around $310 \AA$. An accurate search, which involves comparing observed and predicted line intensities in a $10 \AA$ window, leaves only one possibility, that this is the previously unidentified line in the SERTS-97 active region spectrum (Brosius et al. 2000) at $308.991 \AA$. Lines above $310 \AA$ are excluded by considering quiet-Sun off-limb SOHO/CDS spectra. No strong unidentified lines are present, and there were no indications of significant blending (Del Zanna 1999). The 4-9 transition only accounts for about $1 / 2$ of the intensity of the observed line, in the SERTS-89 spectrum, however its intensity agrees well with the other lines in the SERTS-97 spectrum (see Fig. 5). The intensities of the 4-9 and 16-67 are predicted to be similar, and no other line is present. The energy difference between the experimental and target energies for level 67 is only $2185 \mathrm{~cm}^{-1}$.

The second transition, $21-79$, is predicted to be observable by Hinode/EIS. A similar adjustment to the target energies of all the levels in the $3 \mathrm{~s}^{2} 3 \mathrm{p}^{3} 3 \mathrm{~d}$ configuration predicts a line around $267 \AA$. The only reasonable candidate with the correct wavelength and intensity is the weak line observed at $266.755 \AA$ with Hinode/EIS. The difference between predicted and observed energies is only $1740 \mathrm{~cm}^{-1}$.

The weak 6-103 transition could either be blended with the weak Fe VIII $168.929 \AA$ or be the weak unidentified line at $169.614 \AA$ in the Be76 spectrum. Preference is given to the first choice on energy grounds. The weak 14-54 transition is predicted to be about $1 / 10$ the intensity of the $1-7341.113 \AA$ line at low densities and have a wavelength close to $327 \AA$. There is only one previously unidentified transition, observed by $\mathrm{SOHO} / \mathrm{CDS}$ in quiet-Sun off-limb spectra at $326.3 \AA$, as discussed in Del Zanna (1999). The SERTS-97 wavelength of $326.323 \AA$ is adopted.

\section{Summary and conclusions}

A comprehensive benchmark of Fe XI atomic data against available observations has been presented. All previous line identifications were reviewed by comprehensively comparing the results of the new scattering and structure calculations with experimental data. The results indicate that the new atomic model is accurate to within $10 \%$ for all the strongest transitions, a remarkable result considering the difficulty obtaining a good target for this ion.

Several new levels and transitions have been identified at last, opening the possibility of further identifications along the S I-like sequence, as described in a follow-up paper. The assessment indicates the presence of many blends.

A number of strong lines can now be used to measure electron density reliably. Also, it is shown here for the first time that a few transitions can be used to measure electron temperatures. For the Hinode/EIS wavelengths, Fe XI offers the best diagnostic for coronal temperatures. For lower temperatures, the best diagnostics are from Fe VII and Fe VIII, as described in Del Zanna (2009a) and Del Zanna (2009b). It is recommended that future Hinode/EIS observing sequences include these lines, and that a thorough radiometric calibration programme is put in place in the near future so reliable measurements can be obtained.

Acknowledgements. The support from STFC (Advanced Fellowship and APAPnetwork) is acknowledged. I warmly thank B.C. Fawcett for continuous support and encouragement, and for many exchanges of correspondence over the years. E. Träbert (Bochum and Livermore) is also thanked for useful exchanges of information. Hinode is a Japanese mission developed and launched by ISAS/JAXA, with NAOJ as the domestic partner and NASA and STFC (UK) as international partners. It is operated by these agencies in co-operation with ESA and NSC (Norway).

\section{References}

Badnell, N. R. 1997, J. Phys. B Atom. Mol. Phys., 30, 1

Behring, W. E., Cohen, L., \& Feldman, U. 1972, ApJ, 175, 493

Behring, W. E., Cohen, L., Doschek, G. A., \& Feldman, U. 1976, ApJ, 203, 521 Bromage, G. E., Cowan, R. D., \& Fawcett, B. C. 1977, Phys. Scr, 15, 177

Brosius, J. W., Davila, J. M., \& Thomas, R. J. 1998a, ApJ, 497, L113

Brosius, J. W., Davila, J. M., \& Thomas, R. J. 1998b, ApJS, 119, 255

Brosius, J. W., Thomas, R. J., Davila, J. M., \& Landi, E. 2000, ApJ, 543, 1016

Brown, C. M., Feldman, U., Seely, J. F., Korendyke, C. M., \& Hara, H. 2008, ApJS, 176, 511

Culhane, J. L., Harra, L. K., James, A. M., et al. 2007, Sol. Phys., 60

Del Zanna, G. 1999, Ph.D. Thesis, Univ. of Central Lancashire, UK 
A\&A 514, A41 (2010)

Del Zanna, G. 2009a, A\&A, 508, 501

Del Zanna, G. 2009b, A\&A, 508, 513

Del Zanna, G., \& Ishikawa, Y. 2009, A\&A, 508, 1517

Del Zanna, G., \& Mason, H. E. 2005, A\&A, 433, 731

Del Zanna, G., Bromage, B. J. I., Landi, E., \& Landini, M. 2001, A\&A, 379, 708

Del Zanna, G., Berrington, K. A., \& Mason, H. E. 2004, A\&A, 422, 731

Del Zanna, G., Storey, P. J., \& Mason, H. E. 2010, A\&A, 514, A40

Dere, K. P. 1978, ApJ, 221, 1062

Dere, K. P., Landi, E., Young, P. R., et al. 2009, A\&A, 498, 915

Edlén, B. 1966, Metrologia, 2, 71

Eissner, W., Jones, M., \& Nussbaumer, H. 1974, Comput. Phys. Commun., 8, 270

Fawcett, B. C. 1970, J. Phys. B Atom. Mol. Phys., 3, 1732

Fawcett, B. C. 1971, J. Phys. B Atom. Mol. Phys., 4, 1577

Fawcett, B. C., Gabriel, A. H., \& Saunders, P. A. H. 1967, Proc. Phys. Soc., 89, 863

Feldman, U., \& Doschek, G. A. 1977, J. Opt. Soc. Am., 67, 726

Feldman, U., Behring, W. E., Curdt, W., et al. 1997, ApJS, 113, 195

Gabriel, A. H., Fawcett, B. C., \& Jordan, C. 1966, Proc. Phys. Soc., 87, 825
Gabriel, A. H., Garton, W. R. S., Goldberg, L., et al. 1971, ApJ, 169, 595 Haugan, S. V. H. 1997, SOHO CDS software note, 47 Ishikawa, Y., \& Vilkas, M. J. 2008, Phys. Rev. A, 78, 042501 Jefferies, J. T., Orrall, F. Q., \& Zirker, J. B. 1971, Sol. Phys., 16, 103 Jordan, C. 1971, Sol. Phys., 21, 381

Jupén, C., Isler, R. C., \& Träbert, E. 1993, MNRAS, 264, 627

Malinovsky, L., \& Heroux, M. 1973, ApJ, 181, 1009

Mason, H. E., \& Nussbaumer, H. 1977, A\&A, 54, 547

Nussbaumer, H., \& Storey, P. J. 1978, A\&A, 64, 139

Sandlin, G. D., \& Tousey, R. 1979, ApJ, 227, L107

Sandlin, G. D., Brueckner, G. E., \& Tousey, R. 1977, ApJ, 214, 898

Smitt, R., Svensson, L. Å., \& Outred, M. 1976, Phys. Scr., 13, 293

Storey, P. J., Del Zanna, G., Mason, H. E., \& Zeippen, C. 2005, A\&A, 433, 717

Thomas, R. J., \& Neupert, W. M. 1994, ApJS, 91, 461

Träbert, E. 1998, MNRAS, 297, 399

Wang, J., Marotta, A., \& Datla, R. U. 1984, ApJ, 279, 460

Young, P. R., Landi, E., \& Thomas, R. J. 1998, A\&A, 329, 291

Zeippen, C. J., Seaton, M. J., \& Morton, D. C. 1977, MNRAS, 181, 527 